\title{
On the Blowing Down of Analytic Spaces
}

By

\author{
Akira FUJIKI
}

\section{Introduction}

Let $X$ be a complex space and $A$ be a subspace of $X$. Suppose there exists a proper surjective morphism $f: A \rightarrow A^{\prime}$, where $A^{\prime}$ is another complex space. We say that $X$ can be blown down along $f$, if the following conditions are satisfied; there exists a complex space $X^{\prime}$ containing $A^{\prime}$ as a subspace, and a proper surjective morphism $f^{\prime}: X \rightarrow X^{\prime}$ such that i) $f^{\prime}(A)=A^{\prime}$ and $f^{\prime} \mid A$ coincides with $f$, and ii) $f$ gives an isomorphism of $X-A$ and $X^{\prime}-A^{\prime}$. In this case we say that $\left(X^{\prime}, f^{\prime}\right)$ is the blowing down of $X$ along $f$. Now given a triple $(X, A, f)$ as above, the problem of finding conditions for blowing down $X$ along $f$ has been investigated by many people from various points of view [1] [3] [7] [8] [13] [17] [18] [19] [21] [22] [23] [26].

In this paper we give one sufficient condition for blowing down $X$ under the assumption that $A$ is an effective Cartier divisor on $X$ (Theorem 2). This theorem has been proved by Artin [1] in the category of algebraic spaces. But our method here is a direct generalization of that of [23], and uses a cohomology vanishing theorem for weakly 1-complete complex spaces, which generalizes a similar theoJem of Nakano [24]. (Theorem $\mathrm{N}^{\prime}$ in $\S 1$ ). Then in $\S 2$, we treat the local version of the problem and obtain Theorem 1, the proof of which is the main part of this paper. Next in $\S 3$, we patch together the local blowing downs and obtain a global one. Geometrically, the condition of the theorem says that the normal bundle of $A$ in $X$, when restricted to each fiber of $f$, is sufficiently negative. In $\S 3$ we also show by an example that this 'sufficient' negativity condition is

Received March 19, 1974. Revised May 1, 1974. 
not superfluous, contrary to a conjecture of Markoe and Rossi [21].

The author expresses his hearty thanks to Professor H. Hironaka for valuable suggestions and to Professor S. Nakano for constant encouragement and for leading him to this subject.

\section{§1. Vanishing Theorem on Weakly 1-Complete Complex Spaces}

In this paper complex spaces are not necessarily reduced and have the countable topology. We refer [14] for the conventions and notations about complex spaces, for example; if $A$ is a subspace of a complex space $X$, then $A_{(\mu)}$ denotes the $\mu$-th neighborhood of $A$ in $X$.

Let $V$ be an analytic subspace of a domain $D$ in some $C^{N}$. A $C^{\infty}$ function $\varphi$ on $V$ is by definition the restriction on $V$ of some $C^{\infty}$-function $\tilde{\varphi}$ defined on some neighborhood $W$ of $V$ in $D$. Let $\mathbb{R}$ be reals. Then a $R$-valued $C^{\infty}$-function $\varphi$ on $V$ is said to be strictly plurisubharmonic (resp. plurisubharmonic) on $V$, if there exists a $\tilde{\varphi}$ as above so that it strictly plurisubharmonic (resp. plurisubharmonic) on $W$. In this case we often write briefly as $\varphi$ is s. psh. (resp. psh.) on $V$.

Now let $X$ be a complex space and $L$ be a holomorphic line bundle on $X$. Suppose $L$ is defined by the system of transition functions $\left\{f_{\alpha \beta}\right\}$ with respect to some open covering $\mathfrak{U}=\left\{U_{\alpha}\right\}$ of $X$. A metric on $L$ with respect to this covering is given by the system of positive $C^{\infty}$-functions $h=\left\{h_{\alpha}\right\}$, each defined on $U_{\alpha}$, such that $\left.h_{\beta}\left|h_{\alpha}=\right| f_{\alpha \beta}\right|^{2}$ on $U_{\alpha} \cap U_{\beta}$.

Definition 1. A line bundle $L$ on $X$ is said to be positive (resp. semipositive) if for a suitable choice of $\left\{f_{\alpha \beta}\right\}$ and $\mathfrak{U}=\left\{U_{\alpha}\right\}$ as above, there exists a metric $\left\{h_{\alpha}\right\}$ with respect to $\mathfrak{U}$ such that $-\log h_{\alpha}$ is strictly plurisubharmonic (resp. plurisubharmonic) on $U_{\alpha}$ for every $\alpha$.

Definition 2 [24]. A complex space $X$ is said to be weakly 1-complete, if there exists a real valued $C^{\infty}$-function $\Psi$ on $X$ which is plurisubharmonic and has the property that there exists a $c_{0} \in \mathbb{R} \cup\{\infty\}$ such that $\Psi$ defines a proper $C^{\infty}$-map $X \rightarrow\left(-\infty, c_{0}\right)$. We call $\Psi$ an exhaustion function of $X$, and $c_{0}$ the upper bound of $\Psi$.

Hence in particular $X_{c}=\Psi^{-1}((-\infty, c))$ is relatively compact in $X$ if $c<c_{0}$. But for the given $X$, exhaustion functions are not unique. Hence 
from now on, when we write $X_{c}$ for a weakly 1-complete complex space $X$, we understand that $c<c_{0}$ and $X_{c}=\left\{x \in X ; \Psi_{0}(x)<c\right\}$ for a suitable exhaustion function $\Psi_{0}$ with the upper bound $c_{0}$ on $X$.

The following theorem is proved by Nakano [24].

Theorem N. Suppose $X$ is a weakly 1-complete complex manifold, $E$ is a vector bundle on $X$, and $L$ is a positive line bundle on $X$. Thcn for every $c \in \mathbb{R}$, there exists a positive integer $n_{0}$ such that $H^{i}\left(X_{c}, \mathcal{O}(E) \otimes \mathcal{O}\right.$ $\left.\left(L^{\otimes n}\right) \otimes \mathcal{O}(F)\right)=0$ for $i \geqq 1, n \geqq n_{0}$ and for cvery scmipositive line bundle $F$ on $X$.

In fact, this is a direct consequence of Theorem 2 of [24].

On the other hand, this result can be extended to the following more general situation, using the method of bimeromorphic descent.

Theorem $\mathbb{N}^{\prime 1)}$. Suppose $X$ is a weakly 1-complete complex space and $\mathcal{S}$ is a coherent analytic sheaf on $X$. Let $L$ be a positive line bundle on $X$. Then for every $c \in \mathbb{R}$, there exists a positive integer $n_{0}$ such that $H^{i}\left(X_{c}\right.$, $\left.\mathcal{S} \otimes \mathcal{O}\left(L^{\otimes n}\right) \otimes \mathcal{O}(F)\right)=0$ for $i \geqq 1, n \geqq n_{0}$ and for every semipositive line bundle F on $X$.

Before proceeding to the proof we recall some known results and make preliminary considerations about them.

a) Suppose $X$ is a complex space and $Y$ is a subspace of $X \times \mathbb{P}^{m}$, the direct product of $X$ and a complex projective space $\mathbb{P}^{m}$ of dimension $m$. Let $\mathcal{S}$ be a coherent analytic sheaf on $Y$. Then by a theorem of Grauert and Remmert, for any relatively compact subdomain $D$ of $X$, there exists a positive integer $n_{0}=n_{0}(\mathcal{S}, D)$ such that $R^{i} f_{*}\left(\mathcal{S} \otimes p_{2}^{*} \mathcal{O}_{\boldsymbol{P}^{m}}(n)\right)=0$ on $D$ for $i \geqq 1$ and $n \geqq n_{0}$, where $R^{i} f_{*}$ denotes the $i$-th direct image of sheaves, $p_{2}^{*}$ is induced by the projection to the second factor and $\mathcal{O}(n)$ is the $n$-th tensor product of the fundamental sheaf $\mathcal{O}(1)$ of $\mathbb{P}^{m}$.

b) [14] Suppose $X$ is a complex space and $\mathcal{G}$ is the coherent sheaf of ideals on $X$. Let $f: Y \rightarrow X$ be the monoidal transformation with center $\mathcal{G}$ and set $\mathscr{g}=f^{-1}(\mathcal{G})$. Then for every point $P \in X$, there exists a neighborhood $U \ni P$ such that $f^{-1}(U)$ is realized as a closed subspace of $U \times \mathbb{P}^{m}$ for

1) The author learned the formulation of this theorem and the idea of its proof from Prof. Hironaka. 
some projective space $\boldsymbol{P}^{m}$, such that $f \mid f^{-1}(U)$ is induced by the projection to the first factor and that $\mathscr{g} \cong p_{2}^{*} \mathcal{O}(1)$, where $p_{2}$ and $\mathcal{O}(1)$ are as in a) [14].

Now let $\mathcal{S}_{\mathbf{0}}$ be a coherent analytic sheaf on $Y$ and let $D$ be a relatively compact subdomain of $X$. Then by virtue of a), Leray spectral sequence for the map $f \mid f^{-1}(D)$ gives the following; there exists an integer $n_{0}>0$ such that for every $n \geqq n_{0}$ we have an isomorphism

$$
H^{i}\left(D, f^{*}\left(\mathcal{S}_{0} \otimes g^{n}\right) \otimes \mathcal{O}\left(L_{0}\right)\right) \cong H^{i}\left(f^{-1}(D), \mathcal{S}_{0} \otimes g^{n} \otimes f^{*} \mathcal{O}\left(L_{0}\right)\right) i \geqq 1 \text {, }
$$

where $L_{0}$ is any line bundle on $D$. Here we note the projection formula $f_{*}\left(\mathcal{S}_{0} \otimes g^{n} \otimes f^{*} \mathcal{O}\left(L_{0}\right)\right) \cong f^{*}\left(\mathcal{S}_{0} \otimes g^{n}\right) \otimes \mathcal{O}\left(L_{0}\right)$.

c) Now we write down some exact sequences needed in the sequel. We use the notations in $\mathrm{b}$ ) and assume further that $X$ is reduced and $A$ is nowhere dense in $X$, where $A$ is the subspace of $X$ corresponding to $\mathcal{G}$. Let $\mathcal{S}$ be a coherent analytic sheaf on $X$. Then we have an exact sequence of $\mathcal{O}_{Y}$-modules

$$
0 \longrightarrow \mathscr{I}_{0} \longrightarrow g^{n} \otimes f^{*} \mathcal{S} \stackrel{j}{\longrightarrow} f^{*} \mathcal{S} \longrightarrow f^{*} \mathcal{S} \otimes \mathcal{O}_{Y} / g^{n} \longrightarrow 0
$$

with some torsion sheaf $\mathscr{I}_{0}$, which is obtained by tensoring $f^{*} \mathcal{S}$ with the exact sequence

$$
0 \longrightarrow g^{n} \longrightarrow \mathcal{O}_{Y} \longrightarrow \mathcal{O}_{Y} / g^{n} \longrightarrow 0
$$

Let $\mathscr{M}$ be the image of $j$. Then by taking direct images, we get two exact sequences of $\mathcal{O}_{X}$-modules

$$
\begin{aligned}
& 0 \longrightarrow \mathscr{I}_{1} \longrightarrow f^{*}\left(g^{n} \otimes f^{*} \mathcal{S}\right) \longrightarrow f_{*} \mathscr{M} \longrightarrow \mathscr{I}_{2} \longrightarrow 0 \\
& 0 \longrightarrow f_{*} \mathfrak{M} \longrightarrow f_{*} f^{*} \mathcal{S} \longrightarrow \mathscr{I}_{3} \longrightarrow 0
\end{aligned}
$$

where $\mathscr{I}_{1}, \mathscr{I}_{2}$, and $\mathscr{I}_{3}$ are torsion sheaves. In fact, these sheaves have supports all contained in $A$. On the other hand, the natural map $\alpha_{0}$ : $\mathcal{S} \rightarrow f_{*} f^{*} \mathcal{S}$ gives the following exact sequence

$$
0 \longrightarrow \mathscr{I}^{\prime} \longrightarrow \mathcal{S} \stackrel{\alpha_{0}}{\longrightarrow} f_{*} f^{*} \mathcal{S} \longrightarrow \mathscr{I}^{\prime \prime} \longrightarrow 0
$$

for some torsion sheaves $\mathscr{I}^{\prime}$ and $\mathscr{I}^{\prime \prime}$, whose supports are contained in A.

Next assume that $\mathcal{S}$ is torsion-free so that $\mathscr{I}^{\prime}$ vanishes. Let $\mathcal{A}$ be the 
sheaf of annihilators of $\mathscr{L}^{\prime \prime}$, and $L^{\prime}$ be a line bundle on $X$. Let $\varphi$ be a nonzero section of $\mathcal{O}\left(L^{\prime}\right) \otimes \mathcal{A}$ on an open set $U$. Then we have the following exact sequence

$$
0 \longrightarrow \mathscr{I}_{4} \longrightarrow f_{*} f^{*} \mathcal{S} \stackrel{\alpha}{\longrightarrow} \mathcal{S} \otimes \mathcal{O}\left(L^{\prime}\right) \longrightarrow \mathscr{I}_{5} \longrightarrow 0
$$

on $U$, where $\alpha$ is defined to be the multiplication by $\varphi$, and $\mathscr{I}_{4}$ and $\mathscr{I}_{5}$ are torsion sheaves.

d) For simplicity we use the following terminology. A sheaf of $\mathcal{O}_{X^{-}}$ modules $\mathcal{S}$ on a complex space $X$ is said to be cohomologically q-trivial, or simply, to be $q$-trivial for an integer $q \geqq 0$, if $H^{i}(X, \mathcal{S})=0$ for $i \geqq q$. Then the following fact is easy to see; let

$$
0 \longrightarrow \mathcal{S}_{1} \longrightarrow \mathcal{S}_{2} \longrightarrow \mathcal{S}_{3} \longrightarrow \mathcal{S}_{4} \longrightarrow 0
$$

be an exact sequence of sheaves of $\mathcal{O}_{X}$-modules. Then if $\mathcal{S}_{1}, \mathcal{S}_{2}$ and $\mathcal{S}_{4}$ are q-trivial, then so is $\mathcal{S}_{\mathbf{3}}$.

e) Suppose $X$ is reduced and irreducible and $\mathcal{S}$ is a coherent analytic sheaf on $X$. Let $T_{1}=\{x \in X ; \mathcal{S}$ is not free at $x\}$ and $T_{2}=$ the singular locus of $X$. Then we set $T=T_{1} \cup T_{2}$. This is a nowhere dense analytic subset of $X$. In this situation we can find a coherent sheaf of ideals $\mathcal{G}$ of $\mathcal{O}_{X}$ whose support coincides with $T$, i.e. $\operatorname{Supp}\left(\mathcal{O}_{X} / \mathcal{G}\right)=T$, and which has the following properties; if we let $f: Y \rightarrow X$ be the monoidal transformation with center $\mathcal{G}$, then i) $Y$ is nonsingular and ii) we have an exact sequence of $\mathcal{O}_{Y}$-modules;

$$
0 \longrightarrow \mathscr{I}_{6} \longrightarrow f^{*} \mathcal{S} \longrightarrow \mathcal{O}_{Y}(E) \longrightarrow 0,
$$

where $\mathscr{I}_{6}$ is a torsion part of $f^{*} \mathcal{S}$ and $E$ is some vector bundle on $Y$ [25]. Then tensoring this with an invertible sheaf $g n \otimes f^{*} \mathcal{O}_{X}\left(L^{\otimes m n}\right)$ $\otimes \mathcal{O}_{Y}(F)$, we get an exact sequence

$$
0 \longrightarrow \mathscr{I}_{6} \otimes \mathcal{K} \longrightarrow f^{*} \mathcal{S} \otimes \mathcal{K} \longrightarrow \mathcal{O}_{Y}(E) \otimes \mathcal{K} \longrightarrow 0,
$$

where we put $\mathcal{K}=\mathcal{K}_{m, n}(F)=g^{n} \otimes f^{*} \mathcal{O}_{X}\left(L^{\otimes m n}\right) \otimes \mathcal{O}_{Y}(F)$, and where $\mathcal{g}=f^{-1}(\mathcal{G}), L(\operatorname{resp} . F)$ is a line bundle on $X$ (resp. $Y$ ), and $m, n$ are integers. f) We say that a line bundle $L$ on a complex space $X$ is cohomologically positive on a subdomain $D$ of $X$, if for any coherent analytic sheaf $\mathcal{S}$ on $X$, 
there exists an integer $m_{0}>0$ such that $H^{i}\left(D, \mathcal{S} \otimes \mathcal{O}\left(L^{\otimes m}\right)\right)=0$ for every $m \geqq m_{0}$ and $i \geqq 1$.

Lemma 1. Suppose $X$ is a complex space, $L$ is a line bundle on $X$ and $Q$ is a relatively compact subdomain of $X$. Then $L$ is cohomologically positive on $Q$ if and only if it is cohomologically positive on each irreducible component of $Q_{\text {red }}, Q_{\text {red }}$ being the underlying reduced subspace of $Q$.

Proof is quite analogous to that of [1], Proposition 4.1, 4.2], if we note the following; since $Q$ is relatively compact, we have i) $Q$ has only a finite number of irreducible components, and ii) there exists an integer $m>0$ such that $\Re^{m}=0$ on the whole $Q$, where $\Re$ is the ideal sheaf of $Q_{\text {red }}$.

g) Finally we make the following remark about the behavior of the weakly-l-completeness under holomorphic maps. Suppose $X$ and $Y$ are complex spaces and $f: Y \rightarrow X$ is a morphism. If $X$ is weakly 1-complete with an exhaustion function $\Psi$, then $Y$ becomes naturally a weakly 1complete complex space with an exhaustion function $f^{*} \Psi$, provided that for any $c\left(>c_{0}\right), f^{-1}\left(X_{c}\right)$ is relatively compact. In this case we always take as an exhaustion function of $Y$ the pullback of that of $X$ unless otherwise stated. In particular any closed subspace $T$ of a weakly 1-complete complex space $X$ is naturally weakly l-complete.

Now we come to the proof of the theorem.

Proof of Theorem $N^{\prime}$. Let $\Psi$ be an exhaustion function of $X$ with upper bound $c_{0}$. (Def. 2.) Fix a real number $c>c_{0}$. We denote the restriction of $\mathcal{S}$ on $X$ by $\mathcal{S}_{c}$. Then the proof is by induction on $s=\operatorname{dim}$ $\operatorname{Supp}\left(\mathcal{S}_{c}\right)$, where in general $\operatorname{Supp}\left({ }^{*}\right)$ denotes the support of $*$. Now if $s$ $=0$, then the theorem is trivially true. Thus we assume that $s>0$. First by Lemma 1 we may assume that $X$ is irreducible and reduced. Next, since $H^{i}\left(X_{c}, \mathcal{L} \otimes \mathcal{S}\right)=H^{i}\left(\operatorname{Supp}\left(\mathcal{S}_{c}\right), \mathcal{L} \otimes \mathcal{S}\right)$ for any locally free sheaf $\mathcal{L}$ on $X$, by induction we may take $X_{c}=\operatorname{Supp}\left(\mathcal{S}_{c}\right)$. Moreover we may assume $\mathcal{S}$ is torsion free. For if $T(\mathcal{S})$ denotes the torsion part of $\mathcal{S}$, then $\operatorname{dim} \operatorname{Supp}(T(\mathcal{S}))<\operatorname{dim} X_{c}=\operatorname{dim} X$ and $\mathcal{S} / T(\mathcal{S})$ is torsion free, so that by the exact sequence

$$
0 \longrightarrow T(\mathcal{S}) \longrightarrow \mathcal{S} \longrightarrow \mathcal{S} / T(\mathcal{S}) \longrightarrow 0
$$


and by induction we easily get the assertion.

Now we take $f: Y \rightarrow X$ as in e) with respect to the coherent analytic sheaf $\mathcal{S}$. Then by h), $Y$ is naturally weakly l-complete and $Y_{c}=f^{-1}\left(X_{c}\right)$. To apply Theorem $N$ on $Y_{c}$, we need the following

Lemma 2. For any relatively compact subdomain $D$ of $X$, there exists a positive integer $m_{0}$ such that $J \otimes f^{*} L^{\otimes m}$ is positive on $f^{-1}(D)$ for every $m \geqq m_{0}$, where $J$ is the line bundle corresponding to the invertible sheaf $g$.

Admitting the lemma for a while we continue the proof of the theorem. Take and fix $d \in \mathbb{R}$ with $c_{0}>d>c$. Then by the lemma there exists a positive integer $m_{0}$ such that $J \otimes f^{*}\left(L^{\otimes m}\right)$ is positive on $Y_{d}\left(=f^{-1}\left(X_{d}\right)\right)$ for every $m \geqq m_{0}$. Take and fix such an $m$ for the rest of the proof. Now by h) $Y_{d}$ is weakly l-complete. Hence by Theorem $N$, there exists an integer $n_{1}>0$ such that $\mathcal{O}(E) \otimes\left(g^{m} \otimes f^{*} \mathcal{O}\left(L^{\otimes m n}\right)\right) \otimes \mathcal{O}(F)$ is 1-trivial on $Y_{c}$ for every $n \geqq n_{1}$ and for any semipositive line bundle $F$ on $Y_{d}$. Write $\mathrm{K}_{m, n}(F)=\mathscr{g}^{n} \otimes f^{*} \mathcal{O}\left(L^{\otimes m n}\right) \otimes \mathcal{O}(F)$ as in e). Then, since $\mathscr{I}_{6}$ is torsion in e) and $\mathscr{I}_{6}=\operatorname{Supp}\left(\left.\mathscr{L}_{6}\right|_{Y_{d}}\right)$ is weakly 1 -complete by h), by induction we obtain an integer $n_{2}>0$ such that $\mathscr{L}_{6} \otimes \mathcal{K}_{m, n}(F)$ is 1-trivial for $n \geqq n_{2}$ and for any semipositive line bundle $F$ on $Y_{d}$. Hence by (4) we see immeadiately that $f^{*} \mathcal{S} \otimes \mathcal{K}_{m, n}(F)$ is 1 -trivial on $Y_{c}$ for $n \geqq \max \left(n_{1}, n_{2}\right)$. Now assume that $F$ has the form $F=f^{*}\left(L^{\otimes k} \otimes \bar{F}\right)$ for a $k \geqq 0$ and for a semipositive line bundle $\bar{F}$ on $X_{d}$. This is semipositive as it is the pullback of a semipositive line bundle on $X_{d}$. Then by b) we get an integer $n_{3}>0$ such that:

(A) $f_{*} f^{*} \mathcal{S} \otimes g n \otimes \mathcal{O}\left(L^{\otimes m n+k} \otimes F\right)$ is 1 -trivial for $n \geqq \max \left(n_{1}, n_{2}, n_{3}\right)$ on $X_{c}$.

For such an $n$ we consider exact sequences (1)-(3) in c). For (3) we need

Assertion. Let $\mathcal{A}$ be as in c) i.e. the annihilator of $\mathcal{I}^{\prime \prime}$. Then there exists an integer $s>0$ such that $\mathcal{O}\left(L^{\otimes s}\right) \otimes \mathcal{A}$ has a nonzero section $\psi$ on $X_{c}$.

Proof. In view of Theorem $N$, the arguments similar to those in [18] enable us to get $H^{o}\left(Y_{d}, \mathcal{O}\left(J^{\otimes k} \otimes f^{*} L^{\otimes t k}\right)\right) \neq 0$ for a sufficiently large $k$, 
where $t$ is an integer with $t \geqq m_{0}$ (c.f. Lemma 3 and [24, §3]). Take a $\varphi_{0}(\neq 0) \in H^{0}\left(Y_{d}, \mathcal{O}\left(J^{\otimes k} \otimes f^{*} L^{\otimes t k}\right)\right)$. By the natural isomorphism $H^{0}\left(X_{d}\right.$,

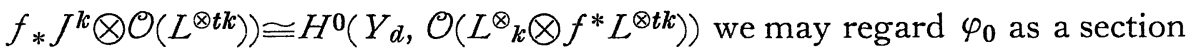
of $\mathcal{O}\left(L^{\otimes t k}\right) \otimes f_{*} g^{k}$ on $X_{d}$. Then since $X_{c}$ is relatively compact in $X_{d}$, we have by the Nullstellensatz an integer $l>0$ such that $\psi=\varphi_{0}^{l} \mid X_{c} \in H^{0}$ $\left(X_{c}, \mathcal{O}\left(L^{\otimes t k}\right) \otimes \mathcal{G k}^{k}\right) . \quad$ On the other hand, since $\operatorname{Supp}\left(\mathcal{O}_{X} / \mathcal{A}\right) \leqq|A|, \mathcal{I}^{k} \subset$ $\mathcal{A}$ on $X_{d}$ for sufficiently large $k$, where $|A|$ denotes the underlying topological space of $A$. Thus we may assume $\psi(\not \equiv) \in H^{0}\left(X_{c}, \mathcal{O}\left(L^{\otimes t k}\right) \otimes \mathcal{A}\right)$ for the $k$ above. This completes the proof.

Thus if we put $U=X_{c}, L^{\prime}=L^{\otimes s}$, and $\varphi=\psi$ in the end of c), then we obtain an exact sequence (3), where $X_{c}, L^{\otimes s}$, and $\psi$ are as above. Then since $L^{\otimes m}$ is positive and $\mathscr{I}_{i}, i=1, \ldots, 5$, are torsion sheaves, again by induction we can find an integer $n_{4}>0$ such that: (B) $\mathscr{I}_{i} \otimes \mathcal{O}\left(L^{\otimes m n} \otimes F_{1}\right)$ is 1-trivial on $X_{c}$ for every $n \geqq n_{4}$, and for any semipositive line bundle $F_{1}$ on $X_{d}$. Now consider the exact sequences $(1)^{\prime}-(3)^{\prime}$ obtained by tensoring the sequences $(1)-(3)$ with $\mathcal{O}\left(L^{\otimes m n+k} \otimes \bar{F}\right)$. Since $L^{\otimes k} \otimes \bar{F}$ is obviously semipositive for $k \geqq 0$, by $(\mathrm{A})$ and $(\mathrm{B})$, the cohomology exact sequences for $(1)^{\prime}-(3)^{\prime}$ together with a remark in d) shows readily that $\mathcal{S} \otimes \mathcal{O}\left(L^{\otimes m n+k+s} \otimes \bar{F}\right)$ is 1 -trivial on $X_{c}$ (for $n \geqq \max _{1 \leqq i \leqq 4} n_{i}$ and $\left.k \geqq 0\right)$. Finally we can find an $n_{0}>0$ such that every $n^{\prime} \geqq \mathrm{n}_{0}$ can be written in the form $n^{\prime}=m n+k+s$ for the fixed $m$ and $s$ and for some $n \geqq \max _{1 \leqq i \leqq 4} n_{i}$ and $k \geqq$ 0 . Thus the theorem is proved.

It remains to prove Lemma 2 .

Proof of Lemma 2. First we make a general remark concerning a metric on a line bundle. Given a holomorphic line bundle $L$ on a complex space $X$, let $\Phi=\left\{\varphi_{0}, \ldots, \varphi_{t}\right\}$ be a finite set of differentiable sections of $L$ which is base point free, namely, for any $x \in X, \varphi_{i}(x) \neq 0$ for some $i$. Suppose $L$ is defined by the system of transition functions $\left\{f_{\alpha \beta}\right\}$ with respect to the covering $\mathfrak{U}=\left\{U_{\alpha}\right\}$ of $X$, and $\varphi_{i}$ are expressed as a system of differentiable functions $\left\{\varphi_{i}, \alpha\right\}$ each defined on $U_{\alpha}$, such that $\varphi_{i, \alpha}=$ $f_{\alpha \beta} \varphi_{i, \beta}$ on $U_{\alpha} \cap U_{\beta}$. Then if we put $h_{\alpha}=1 / \sum_{i=0}^{t}\left|\varphi_{i \alpha}\right|^{2}, h_{\alpha}$ are positive $C^{\infty}$-functions defined on $U_{\alpha}$ and satisfies the relation $\left.h_{\beta}\left|h_{\alpha}=\right| f_{\alpha \beta}\right|^{2}$ on $U_{\alpha} \cap U_{\beta}$. Thus these $h_{\alpha}$ gives a metric on $L$. We use this remark in 
constructing a metric on the line bundle $J$. Let $\mathcal{A}_{X}$ (resp. $\mathcal{A}_{Y}$ ) be the sheaf of germs of differentiable functions on $X$ (resp. $Y$ ). Since $X$ is paracompact and $D$ is relatively compact, there exists a finite set $\Phi_{0}$ of elements of $H^{0}\left(X, \mathcal{A}_{X} \otimes \mathcal{O}_{X} f_{*}(\mathcal{O}(J))\right.$ which generates each stalk of $\mathcal{A}_{X} \otimes$ $f_{*} \mathcal{O}(J)$ on the closure $\bar{D}$ of $D$ (c.f. [15] ). Then it is easily seen that this $\Phi_{0}$ in turn gives a set $\Phi$ of elements of $H^{0}\left(Y, \mathcal{A}_{Y} \otimes \mathcal{O}_{Y} \mathcal{O}(J)\right)$ which are holomorphic along each fiber of $f$ and which has no base points on $f^{-1}$ $(\bar{D})$. Hence by the above remark, we have a metric $h$ on $J$ over $f^{-1}(\bar{D})$, which has the additional property that, when restricted to each fiber of $f$ it coincides with the restriction of the standard metric of the hyperplane bundle of the projective space, where a fiber $f^{-1}(P), P \in X$, is regarded as a subspace of a projective space as in b). So if $h=\left\{h_{\alpha}\right\}$ with respect to a suitable covering $\mathfrak{U}=\left\{U_{\alpha}\right\}$ of $D$, then in particular - $\log h_{\alpha}$ is strictly plurisubharmonic with respect to the local coordinates of fibers of $f$ in $U_{\alpha}$. More precisely, let $\mathfrak{B}=\left\{V_{\iota}\right\}$ be a finite covering of the closure $\bar{D}$ of $D$ such that $f^{-1}\left(V_{\imath}\right)$ is embedded in $V_{\iota} \times \mathbb{P}^{n}$ (c.f. b). Then we can take a covering $\mathfrak{U}=\left\{U_{\alpha}\right\}$ of $f^{-1}(\bar{D})$ with the following properties; 1$) \mathfrak{U}$ is a refinement of the covering $f^{-1}(\mathfrak{B})$ so that each $U_{\alpha}$ is embedded as a subspace of $V_{\alpha}^{\prime} \times G_{\alpha}$, where $V_{\alpha}^{\prime}$ (resp. $G_{\alpha}$ ) is the subdomain of $V_{\iota(\alpha)}$ (resp. $\left.\mathbb{P}^{n}\right)$ and $G_{\alpha}$ has the coordinate system $\left.\left(w_{i}^{\alpha}\right), 2\right) h_{\alpha}$ is the restriction of a $\mathbb{R}$-valued $C^{\infty}$-function $\tilde{h}_{\alpha}$ on $V_{\alpha}^{\prime} \times G_{\alpha}$ which is holomorphic with respect to the coordinates $\left(w_{i}^{\alpha}\right)$, and 3 ) the hessian $-\frac{\partial^{2} \log \tilde{h}_{\alpha}}{\partial w_{i}^{\alpha} \partial \bar{w}_{j}^{\alpha}}$ of $-\log \tilde{h}_{\alpha}$ with respect to the coordinate system $\left(w_{i}^{\alpha}\right)$ is positive definite at every point of $V_{\alpha}^{\prime} \times G_{\alpha}$. Then, since $L$ is positive on $X$ and $\bar{D}$ is compact, there exists an integer $m_{0}$ such that $\mathscr{g} \otimes f^{*}\left(L^{m}\right)$ is positive for $m \geqq m_{0}$ on $D$ (c.f. [22, Chap II, Lemma 4] ).

This lemma could be regarded as a direct generalization of [18, Theorem 8] together with its proof to the general projective morphisms.

Remark. The above method of bimeromorphic descent can be applied (with slight modifications), for instance to deduce the finiteness theorem of cohomology on compact complex spaces with coefficients in coherent analytic sheaves from that on compact complex manifolds with coefficients in locally free sheaves. 
Now a line bundle $L$ on a complex space $X$ is said to be ample on a subdomain $D$ of $X$, if there exists an integer $m_{0}>0$ such that for any $m \geqq m_{0}$, we can find a finite number of elements $\varphi_{0}, \ldots, \varphi_{t}$ of $H^{0}\left(D, O\left(L^{\otimes m}\right)\right)$ which embedds $D$ as a locally closed subspace of a projective space $\mathbb{P}^{t}$ such that $\Phi^{*}(\mathcal{O}(1))=\mathcal{O}\left(L^{\otimes m}\right)$, where $\Phi: D G \mathbb{P}^{t}$ is the associated map.

Lemma 3. Suppose $X$ is a weakly 1-complete complex space and $L$ is a line bundle on $X$. Then the following four conditions are equivalent for every $c \in \mathbb{R}$.

1) $L$ is ample on $X_{c}$.

2) For any coherent analytic sheaf $\mathcal{S}$ on $X$, there exists an integer $m_{1}$ such that for every $m \geqq m_{1} S \otimes \mathcal{O}\left(L^{\otimes m}\right)$ is generated on $X_{c}$ by a finite number of elements of $H^{0}\left(X_{c}, S \otimes \mathcal{O}\left(L^{\otimes m}\right)\right)$.

3) $L$ is cohomologically positive on $X_{c}$.

4) $L$ is positive on $X_{c}$.

The equivalence of 1)-3) can be proved quite analogously to [1], Theorem 3.1]. That 4) $\rightarrow 3$ ) follows from Theorem $N^{\prime}$. Finally, if $L$ is ample on $X_{c}$, then $L^{\otimes m}$ is positive on $X_{c}$ for all sufficient large $m$, since it can be regarded as the restriction on $X_{c}$ of a hyperplane bundle of the ambient projective space. From this we conclude that $L$ is positive on $X$. Hence $1) \rightarrow 4$ ).

\section{§2. Local Blowing Down}

Let $X$ be a complex space. Assume that $X$ is holomorphically convex. Then it is known by the theory of Remmert quotients that $X$ admits a proper surjective morphism $F: X \rightarrow X^{\prime}$ with $X^{\prime}$ a stein space. (See [28] for the nonreduced case). On the other hand, by the theory of embedding a stein space into a number space ([27] for the nonreduced case), there exists a proper morphism $j: X^{\prime} \subseteq \mathbb{C}^{N}$ of $X^{\prime}$ into some complex number space $\mathbb{C}^{N}$. Let $\left(t_{1}, \ldots, t_{N}\right)$ be the coordinates of $\mathbb{C}^{N}$ and put $g=$ $j \cdot F$. Then we define a $\mathbb{R}$-valued $C^{\infty}$-function $\Psi$ on $X$ as the pullback by $g$ of the function $\sum_{i=1}^{N}\left|t_{i}\right|^{2}$ on $C^{N} ; \Psi=g^{*}\left(\sum_{i=1}^{N}\left|t_{i}\right|^{2}\right)$. Clearly $\Psi$ is plurisubharmonic and defines a proper map $\Psi: X \rightarrow \mathbb{R}$. Hence $X$ is weakly 1 complete with an exhaustion function $\Psi$. From now on, when we are given 
a holomorphically convex space $X$, we regard it as a weakly l-complete complex space by choosing and fixing once and for all the maps $F$ and $j$, and defining an exhaustion function $\Psi$ as above. In particular we use freely the notations such as $X_{c}, c \in R$, for a holomorphically convex space $X$.

Now in general a subspace $A$ of complex space $X$ is said to be an effective cartier divisor, if at each point of $X$ the ideal sheaf $\mathcal{I}_{A}$ of $A$ in $X$ is generated by a single element which is not a zero divisor. In this section we shall prove

Theorem 1. Suppose $X$ is a complex space and $A$ is an effective cartier divisor on $X$. Let $L=[A]$ be the corresponding line bundle. We assume the following conditions:

1) $A$ is holomorphically convex.

2) The restriction $L_{A}^{*}$ of $L^{*}$ onto $A$ is positive, where $L^{*}$ is the dual line bundle of $L .{ }^{11}$

3) $H^{1}\left(A, O\left(L_{A}^{* \mu}\right)\right)=0$ for every $\mu>0$.

Then for every $c \in \mathbb{R}$ there exists a neighborhood $U_{c}$ of $A_{c}$ in $X$ with $U_{c} \cap A=A_{c}$ such that $U_{c}$ admits a proper morphism $h: U_{c} \rightarrow C^{l}$ which is isomorphic outside $A_{c}$.

For the proof we need the following two lemmas.

Lemma 4. Suppose $X$ is a complex space and $A$ is an effective cartier divisor on $X$. Let $L=[A]$ be the corresponding line bundle. We assume the following conditions;

1) $A$ is weakly 1-complete, and

2) the restriction $L_{A}^{*}$ of $L^{*}$ onto $A$ is positive.

Then for every $c \in \mathbb{R}$ there exists a neighborhood $W_{c}$ of $A_{c}$ in $X$ with $W_{c} \cap A=A_{c}$ which has the following properties;

i) $L^{*}$ is positive on $W_{c}$.

ii) There exists a plurisubharmonic function $\Psi$ on $W_{c}$ which is strictly plurisubharmonic on $W_{c}-A_{c}$.

Lemma 5. Under the notations and assumptions of Theorem 1,

1) Here the restriction means the analytic restriction. Thus $L_{A}^{*}=i^{*} L^{*}, i: A \subseteq X$ being the inclusion. 
there exists for every $c \in \boldsymbol{R}$ an arbitrary small neighborhood $U_{c}$ of $A_{c}$ in $X$ with $U_{c} \cap A=A_{c}$ which is weakly 1-complete.

We first prove the theorem using these lemmas.

Proof of the theorem. Take and fix an arbitrary $c \in \mathbb{R}$ and choose $\mathrm{c}_{1}, \mathrm{c}_{2} \equiv \boldsymbol{R}$ such that $c<c_{1}<c_{2}$. Then by Lemma 4 there exists a neighborhood $V$ of $A_{c_{2}}$ in $X$ such that $L^{*}$ is positive on $V$. Then by Lemma 5 we can find a neighborhood $W$ of $A_{c_{1}}$ in $X$ which is weakly 1-complete and is contained in $V$. Then by Lemma 3 , since $L^{*}$ is positive on $W$, we infer that for any relatively compact subdomain $Q$ of $W$, there exists an integer $m>0$, and a finite number of elements $\varphi_{0}, \ldots, \varphi_{n} \in H^{0}(W$, $\left.\mathcal{O}\left(L^{* \otimes m}\right)\right)$ such that i) the associated map $\Phi=\left(\varphi_{0}: \ldots: \varphi_{n}\right)$ of $X$ into a projective space $\boldsymbol{P}^{n}$ gives a biholomorphic embedding of $Q$ onto a locally closed analytic subspace of $\boldsymbol{P}^{n}$ and that ii) $\Phi^{*}(\mathcal{O}(1))=\mathcal{O}\left(L^{* \otimes m}\right)$ on $Q$. In particular $\varphi_{i}$ generate each stalk of $L^{* \otimes m}$ on $Q$. Now we take and fix $Q$ which containes the closure of $A_{c}$ in $X$. In view of the natural isomorphism $\iota: \mathcal{O}\left(L^{* \otimes m}\right) \cong \mathcal{G}_{A}^{m}$, defined locally by the multiplication of the $m$-th power of the local equation of $A$ in $X$, we may regard $\varphi_{i}$ as elements of $H^{0}\left(W, \mathcal{G}_{A}^{m}\right)$. We denote these by $\bar{\varphi}_{i}$ in distinction from $\varphi_{i}$. Then $\bar{\varphi}_{i}$ define a holomorphic map $\bar{\Phi}: Q \rightarrow C^{n+1}$ by $\bar{\Phi}(x)=\left(\bar{\varphi}_{0}(x), \ldots, \bar{\varphi}_{n}(x)\right)$. Then $\varphi_{i}$ generating each stalk is easily seen to be equivalent to the fact that $\bar{\Phi}^{-1}(0)=Q \cap A$ (set theoretically). In particular if we put $\bar{\varphi}=$ $\sum_{i=0}^{n}\left|\bar{\varphi}_{i}\right|^{2}$, then $\bar{\varphi} \neq 0$ for any $x \in Q-Q \cap A$.

Now take some $W_{d}$ for a weakly l-complete $W$ which contains $Q$. By the vanishing theorem there exists an integer $m_{1}>0$ such that $H^{1}\left(W_{d}\right.$, $\left.\mathcal{G}_{A}^{m}\right)=0$. Hence by virtue of the exact sequences

$$
0 \longrightarrow \mathcal{I}_{A}^{m} \longrightarrow \mathcal{O}_{X} \longrightarrow \mathcal{O}_{X} / \mathcal{I}_{A}^{m} \longrightarrow 0,
$$

we get a surjective map $\rho: H^{0}\left(W_{d}, \mathcal{O}_{w}\right) \rightarrow H^{0}\left(A \cap W_{d}, \mathcal{O}_{w} / \mathcal{J}_{A}^{m_{1}}\right)$. On the other hand, from the exact sequences

$$
0 \longrightarrow \mathcal{O}_{A}\left(L_{A}^{* \mu}\right) \longrightarrow \mathcal{O}_{X} / \mathcal{I}_{A}^{\mu}+1 \longrightarrow \mathcal{O}_{X} / \mathcal{I}_{A}^{\mu} \longrightarrow 0 \quad \mu=1,2, \ldots
$$

and from 3) of the theorem, it follows that $\rho_{\mu}: H^{0}\left(A, \mathcal{O}_{X} / \mathcal{J}_{A}^{\mu+1}\right) \rightarrow H^{0}(A$, $\left.\mathcal{O}_{A}\right)$ is surjective for every $\mu>0$. Thus we can find for any $f \in H^{0}\left(A, \mathcal{O}_{A}\right)$ an element $\tilde{f} \in H^{0}\left(W_{d}, \mathcal{O}_{W}\right)$ such that $\tilde{f}|Q \cap A=f| Q \cap A$. 
Now let $f_{i}, i=1, \ldots, N$, be the elements of $H^{0}\left(A, \mathcal{O}_{A}\right)$ which define a proper holomorphic map $F: A \rightarrow C^{N}$ into some number space $\mathbb{C}^{N}=C^{N}$ (t) so that $A_{c}=\left\{\sum_{i=1}^{N}\left|f_{i}\right|^{2}<c\right\}$ (see the beginning of this section). Then by the above, there exist elements $\tilde{f}_{i} \in H^{0}\left(W_{d}, \mathcal{O}_{W}\right)$ which extends $f_{i} \mid Q \cap A$. Let $F: Q \rightarrow C^{N}$ be the map defined by these $\tilde{f}_{i}$. Then we get finally the following commutative diagram

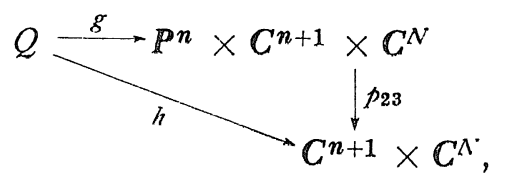

where $g=\Phi \times \bar{\Phi} \times F, h=\bar{\Phi} \times F$, and $p_{23}$ is the projection to the second and the third factors. Note that by the definition of $\Phi$ and $\Phi, g$ factors through $g^{\prime}: Q \rightarrow Y$ and $j: Y \hookrightarrow \mathbb{P}^{n} \times C^{n+1} \times C^{N}$, where $Y$ is the monoidal transform of $C^{n+1} \times C^{N}$ with center $D=0 \times C^{N}$, and $j$ is the inclusion. Explicitly, $Y$ is a submanifold of $\boldsymbol{P}^{n} \times \boldsymbol{C}^{n+1} \times C^{N}$ defined by the system of equations $\xi_{i} z_{i}=z_{i} \xi_{j}, 0 \leqq i, j \leqq n$, where $\left(\xi_{i}\right)\left(\operatorname{resp} .\left(z_{i}\right)\right)$ is the homogeneous coordinates of $\boldsymbol{P}^{n}$ (resp. the coordinates of $C^{n+1}$ ). Further $\sigma=\left.p_{23}\right|_{y}$ coincides with the monoidal transformation with center $D$. Thus we have $h=$ $\sigma \cdot g^{\prime}$. Let $B=\sigma^{-1}(D)$. Then $B \cap g(Q)=g(A \cap Q)$. Indeed, as noted above, $x \in A$ if and only if $\bar{\Phi}(x)=0$. Hence $h(x) \in D$ if and only if $x \in A$. This gives the above relation on account of $h=\sigma g^{\prime}$. Since $\sigma$ is isomorphic outside $B$ and $g$ is an embedding, we infer from this that $h$ is isomorphic outside $A \cap Q$. Now define a subdomain $Q_{\varepsilon, b}$ of $Q$ by $Q_{\varepsilon, b}=\{x \in Q$; $\left.\left.\left|\bar{\varphi}_{i}(x) !<\varepsilon, \tilde{f}=\sum_{j=1}^{N}\right| \tilde{f}_{i}\right|^{2}<b\right\}, \varepsilon, b>0$. Then it is easily checked that for every $b$, with $c \geqq b>0, Q_{\varepsilon, b}$ is relatively compact in $Q$ if we take $\varepsilon$ sufficiently small. From this we infer readily that the restricted map $h \mid Q_{\varepsilon, c}$ : $Q_{\varepsilon, c} \rightarrow D_{\varepsilon} \times D_{c}$ is proper for $\varepsilon$ sufficiently small, where $D_{\varepsilon}=\left\{\left|z_{i}\right|<\varepsilon\right\}$ and $D_{c}=\left\{\sum_{j=1}^{N}\left|t_{j}\right|^{2}<c\right\}$. Put $U_{c}=Q_{\varepsilon, c}$. Then, since $D_{\varepsilon} \times D_{c}$ is stein and hence can be embedded into some number space $C^{l}$, it is clear that this $U_{c}$ has the desired properties of the theorem.

Q.E.D.

We now give the proofs of Lemma 4 and 5 .

Proof of Lemma 4. First we choose and fix an arbitrary $c \in \mathbb{R}$. Since $A$ is weakly 1 -complete and $L_{A}^{*}$ is positive, we may apply the vanish- 
ing theorem to the pair $\left(A, L_{A}^{*}\right)$. Thus for any $d \in \mathbb{R}, L_{A_{d}}^{*}$ is cohomologically positive. Then by Lemma 1 for every $\nu>0 L_{d, \nu}^{*}=L_{A_{d, \nu}}^{*}$ also is cohomologically positive on $A_{d, \nu}$, where $A_{d, \nu}$ is the $\nu$-th neighborhood of $A_{\boldsymbol{d}}$ in a suitable neighborhood $U$ of $A_{d}$ in $X$. Now we take and fix first $d \in \mathbb{R}$ such that $d>c$ and then $\nu \geqq 2$ so large that $H^{1}\left(A_{d}, \mathcal{O}\left(L_{A}^{* \otimes \mu}\right)\right)$ $=0$ for every $\mu \geqq \nu$. For these $d$ and $\nu$, by Lemma 3 there exists a positive integer $m=m(d, \nu)$ and a finite number of elements $\varphi_{0}, \ldots$, $\varphi_{t} \in H^{0}\left(A_{d, \nu}, \mathcal{O}\left(L_{d, \nu}^{* \otimes m}\right)\right)$, such that the associated map $\Phi_{0}: A_{d, \nu} \rightarrow \mathbb{P}^{t}$ of $A_{d, \nu}$ into a projective space $\mathbb{P}^{t}$ gives a biholomorphic embedding of $A_{c, \nu}$ onto a locally closed analytic subset $B_{c}$ of $\mathbb{P}^{t}$, and further that there exists an isomorphism of line bundles $\gamma_{\nu}: \Phi_{0}^{*}(H) \simeq L_{A}^{*}$ on $A_{c, \nu}$, where $A_{c, \nu}=A_{d, \nu} \mid A_{c}$ and $H$ is the hyperplane bundle on $\mathbb{P}^{t}$. For simplicity, from now on, we suppress the suffix $d$ so that for example we write $A_{\nu}$ and $L_{\nu}$ instead of $A_{d, \nu}$ and $L_{d, \nu}$ respectively.

Now our next purpose is to extend these $\varphi_{i}$ first to the sections $\varphi_{i}^{(\mu)}$ of $H^{0}\left(A_{\mu}, \mathcal{O}\left(L_{\mu}^{* \otimes m}\right)\right)$ for sufficiently large $\mu$, and then extend these $\varphi_{i}^{(\mu)}$ to the $C^{\infty}$-sections $\bar{\varphi}_{i}$ of $L^{* \otimes^{m}}$. For the first step we observe the following exact sequence of cohomology

$$
\begin{aligned}
\longrightarrow & H^{0}\left(A_{\mu}, \mathcal{O}\left(L_{\mu}^{* \otimes^{m}}\right)\right) \stackrel{a_{\mu}}{\longrightarrow} H^{0}\left(A_{\mu-1}, \mathcal{O}\left(L_{\mu-1}^{* \otimes m}\right)\right) \\
& \longrightarrow H^{1}\left(A, \mathcal{G}_{A}^{\mu}{ }_{A} \mathcal{G}_{A}^{\mu+1} \otimes \mathcal{O}_{A}\left(L_{A}^{* \otimes m}\right)\right)
\end{aligned}
$$

which comes from the exact sequence of $\mathcal{O}_{X}$-modules

$$
\begin{aligned}
0 \longrightarrow \mathcal{G}_{A}^{\mu} / \mathcal{G}_{A}^{\mu+1} \otimes \mathcal{O}\left(L^{* \otimes m}\right) & \longrightarrow \mathcal{O}_{X} / \mathcal{G}_{A}^{\mu+1} \otimes \mathcal{O}\left(L^{* \otimes^{m}}\right) \\
& \longrightarrow \mathcal{O}_{X} \mid \mathcal{G}_{A}^{\mu} \otimes \mathcal{O}\left(L^{* \otimes m}\right) \longrightarrow 0
\end{aligned}
$$

Then since $H^{1}\left(A, \mathcal{G}_{A}^{\mu} / \mathcal{G}_{A}^{\mu+1} \otimes \mathcal{O}\left(L^{* \otimes m}\right)\right) \cong H^{1}\left(A, \mathcal{O}\left(L_{A}^{* \otimes m+\mu}\right)\right)=0$ by the choice of $\nu, a_{\mu}$ is surjective for every $\mu \geqq \nu$. Hence we can extend $\varphi_{i}$ successively to the elements $\varphi_{i}^{(\mu)}$ of $H^{0}\left(A_{\mu}, \mathcal{O}\left(L_{\mu}^{* \otimes m}\right)\right)$ for arbitrary large $\mu$. Next we fix one such $\mu$, which is to be determined in the sequel, and consider the following commutative diagram of exact sequences

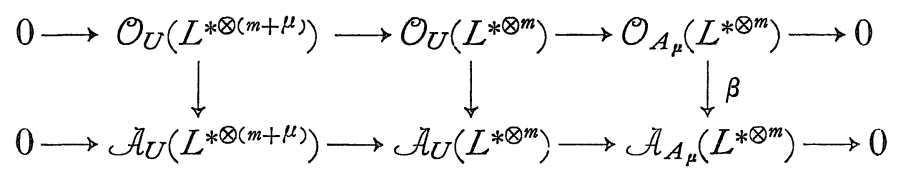

where $\mathcal{A}\left({ }^{*}\right)$ denotes the sheaf of germs of differentiable sections of cor- 
responding line bundles, and the vertical arrows map holomorphic sections into $C^{\infty}$-sections. Since $U$ is paracompact and therefore $\mathcal{A}_{U}\left(L^{* \otimes(m+\mu)}\right)$ is fine, we see that $H^{1}\left(U, \mathcal{A}\left(L^{* \otimes(m+\mu)}\right)\right)=0$ [15]. Hence we have a surjective map $\alpha: H^{0}\left(U, \mathcal{A}\left(L^{* \otimes^{m}}\right)\right) \rightarrow H^{0}\left(\mathcal{A}_{\mu}, \mathcal{A}\left(L_{\mu}^{* \otimes^{m}}\right)\right)$. Now let $\beta_{*}: H^{0}$ $\left(U, \mathcal{O}\left(L^{* \otimes^{m}}\right)\right) \rightarrow H^{0}\left(U, \mathcal{A}\left(L^{* \otimes^{m}}\right)\right)$ be induced by $\beta$ and let $\tilde{\varphi}_{i}^{(\mu)}=\beta_{*}\left(\tilde{\varphi}_{i}^{(\mu)}\right)$. Take for each $\tilde{\varphi}_{i}^{(\mu)}$ an element $\tilde{\varphi}_{i} \in H^{0}\left(U, A\left(L^{* \otimes m}\right)\right)$ such that $\tilde{\varphi}_{i}^{(\mu)}=\alpha$ $\left(\tilde{\varphi}_{i}\right)$. These $\tilde{\varphi}_{i}$ are the desired elements.

Using these $\tilde{\varphi}_{i}$ we define a $C^{\infty}$-map $\Phi: U \rightarrow \mathbb{P}^{t}$ of $U$ into a projective space $\mathbb{P}^{t}$ as usual. It is clear that $\Phi \mid A_{\nu}=\Phi_{0}$. Further, since $\nu \geqq 2$ and $\Phi_{0}$ is biholomorphic on $A_{c}$, , we can find a neighborhood $V$ of $A_{\boldsymbol{c}, \nu}$, in $X$ such that $\Phi$ defines a $C^{\infty}$-embedding of $V$ onto a locally closed $C^{\infty}$ subspace of $\mathbb{P}^{t}$, and that there exists an $C^{\infty}$-isomorphism $\tilde{\gamma}: \Phi^{*}(H) \simeq$ $L^{* \otimes^{m}}$ on $U$ which induces the biholomorphic isomorphism $\gamma_{\mu}$ when restricted to $A_{\mu}$. Let $\left(\xi_{0}: \ldots: \xi_{t}\right)$ be the homogeneous coordinates of $\mathbb{P} t, V_{\alpha}^{(0)}=\left\{\xi_{\alpha} \neq 0\right\}$ and $V_{\alpha}=\Phi^{-1}\left(V_{\alpha}^{(0)}\right)$. Then by the above isomorphism we may assume the following situation; $L^{* \otimes m}$ is defined by the system of transition functions $\left\{f_{\alpha \beta}\right\}$ with respect to the covering $\left\{V_{\alpha}\right\}$ of $V$, such that $f_{\alpha \beta}=\Phi^{*}\left(\xi_{\beta} / \xi_{\alpha}\right)$ on $V_{\alpha} \cap V_{\beta}$. Moreover the sections $\varphi_{i}$ are given by the system of $C^{\infty}$-functions $\left\{\tilde{\varphi}_{i, \alpha}\right\}$, each defined on $V_{\alpha}$ by $\tilde{\varphi}_{i, \alpha}=$ $\Phi^{*}\left(\xi_{i} \mid \xi_{\alpha}\right)$. Now put $a_{\alpha}=\sum_{i=0}^{t}\left|\tilde{\varphi}_{i \alpha}\right|^{2}=\Phi^{*}\left(\left.\sum_{i=0}^{t}\left|\xi_{i}\right| \xi_{\alpha}\right|^{2}\right)$. Then $1 / a_{\alpha}$ define a metric of $L$ which is the pullback by $\Phi$ of the natural metric of the hyperplane bundle of $\mathbb{P}^{t}$. Hence for (i) it is sufficient to show that $\log a_{\alpha}$ is strictly plurisubharmonic at each point of $V \cap A$.

For this, take an arbitrary point $x \in A_{c}$ and let $y=\Phi(x)$. We may assume that $y \in V_{0}^{(0)}$. Then we have the following local situation; i) there exists a neighborhood $U$ of $x$ in $X$ and an isomorphism $\tau: U \simeq B$ $\subseteq D \subseteq \mathbb{C}^{l}$ of $U$ onto an analytic subset $B$ of a domain $D$ in $\mathbb{C}^{l}$ such that $\tau(x)=0$. ii) $\Phi \mid U$ extends to a $C^{\infty}$-embedding $\Phi^{\prime}$ of $D$ into $V_{0}$ which is naturally isomorphic to $C^{t}$. iii) If we denote $\Phi^{\prime}$ by $(t+1)$-ple of $C^{\infty}$ -

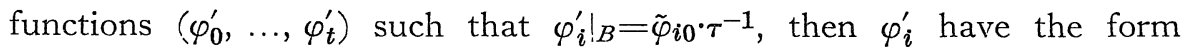
$\varphi_{i}^{\prime}=\varphi_{i}^{(1)}+\varphi_{i}^{(2)} f^{\prime \mu}$, where $\varphi_{i}^{(1)}$ and $f^{\prime}$ (resp. $\varphi_{i}^{(2)}$ ) are holomorphic functions (resp. $C^{\infty}$-functions) on $D$, and $\left.f^{\prime}\right|_{B}$ is a local equation of $\tau(A)$ in $B$. To see ii) and iii), first we take $U$ so small that there exists a local equation $f$ of $A$ on $U$. Then by construction $\tilde{\varphi}_{i, 0}$ can be written as $\tilde{\varphi}_{i, 0}=\tilde{\varphi}_{i}^{(1)}$ 
$+\tilde{\varphi}_{i}^{(2)} f^{\mu}$, where $\tilde{\varphi}_{i}^{(1)}$ (resp. $\tilde{\varphi}_{i}^{(2)}$ ) are holomorphic (resp. $C^{\infty}$ ) functions on $U$ such that $\tilde{\varphi}_{i}^{(1)}$ give a biholomorphic embedding of $U_{(\mu)}$ into a locally closed analytic subspace of $V_{0}^{(0)}$. Then we have only to extend $\tilde{\varphi}_{i}^{(1)} \cdot \tau^{-1}$ and $f \cdot \tau^{-1}$ (resp. $\tilde{\varphi}_{i}^{(2)} \cdot \tau^{-1}$ ) to holomorphic (resp. $C^{\infty}-$ ) functions $\varphi_{i}^{(1)}$ and $f^{\prime}$ (resp. $\left.\varphi_{i}^{(2)}\right)$ on $D$. Hence our task is now reduced to show that hessian $\frac{\partial^{2} \log \tilde{a}_{\alpha}}{\partial w^{i} \partial \bar{w}^{j}}$ of the function $\log \tilde{a}_{\alpha}, \tilde{a}_{\alpha}=\sum_{i=0}^{t}\left|\varphi_{i}^{\prime}\right|^{2}$, with respect to the coordinates $\left(w^{i}\right)$ of $C^{l}$ is positive definite at the origin. But this follows easily from $\mu \geqq 2$. (See the proof of Lemma 6 below, putting $k=0$ there.)

Next we shall show ii). First recall the isomorphism $\mathcal{O}_{X}\left(L^{*}\right) \simeq \mathcal{I}_{A}$ and the $C^{\infty}$-isomorphisms $\gamma_{\alpha}: \mathcal{O}_{V \alpha}\left(L^{* \otimes^{m}}\right) \simeq \mathcal{O}_{V \alpha}$ (which is analytic on $V_{\alpha} \cap A_{\mu, c)}$. In view of these isomorphisms, we infer readily that there exists a system of $C^{\infty}$-functions $\left\{f_{\alpha}\right\}$, each defined on $V_{\alpha}$, with the following property; i) for every point $P \in V_{\alpha}$ we can find a neighborhood $U_{P}$ of $P$ in $X$ such that $\left.f_{\alpha}\right|_{U_{P}}$ can be written in the form $\left.f_{\alpha}\right|_{U_{P}}=\lambda_{P} f_{P}$, where $f_{P}$ is a local equation of $A$ in $U_{P}$, and $\lambda_{P}$ is a non-vanishing $C^{\infty}$-function on $U_{P}$ which is holomorphic when restricted to $A_{\mu} \cap U_{P}$, and ii) $f_{\alpha}^{m} /$ $f_{\beta}^{m}=\varphi^{*}\left(\xi_{\beta} / \xi_{\alpha}\right)$ on $V_{\alpha} \cap V_{\beta}$. Then we can define a $\boldsymbol{R}$-valued $C^{\infty}$-function $\Psi$ globally defined on $W_{d}$ by $\Psi=\left|f_{\alpha}\right|^{2 m}\left(\sum_{i=0}^{t}\left|\tilde{\varphi}_{i \alpha}\right|^{2}\right)$ on $V_{\alpha}$. We contend that this $\Psi$ satisfies the properties ii) of the lemma. For this, again fix a point $x \in A_{c}$ and go back to the local situation described above. Then, by the same argument as above we can take $U$ such that it has further properties that $\Psi \cdot \tau^{-1}$ is the restriction of the function $\Psi_{D}$ on $D$ of the form $\Psi_{D}=\left|\lambda_{D} f^{\prime}\right|^{2 m}\left(\sum_{i=1}^{n}\left|\varphi_{i}^{\prime}\right|^{2}\right)$, where $\lambda_{D}$ is a $C^{\infty}$-function on $D$ written as $\lambda_{D}=\lambda^{(1)}+f^{\prime \mu} \lambda^{(2)}$ with $\lambda^{(1)}$ (resp. $\lambda^{(2)}$ ) holomorphic (resp. $C^{\infty}$ ) on $D$. Now we have to show this is plurisubharmonic on a suitable neighborhood $U^{\prime}$ of the locus $A^{\prime}$ of $f^{\prime}=0$ in $D$, and strictly plurisubharmonic on $U^{\prime}-A^{\prime}$, (if we take $\mu$ sufficiently large). But this follows from the following lemma by the definition of $\lambda_{D}$.

Q.E.D.

Lemma 6. Suppose $\varphi_{i}^{(1)}, 1 \leqq i \leqq n$, and $f$ are holomorphic functions and $\varphi_{i}^{(2)}, 1 \leqq i \leqq n$, are $C^{\infty}$-functions on a domain $D$ of $C^{l}=C^{l}\left(z_{1}, \ldots\right.$, $\left.z_{l}\right)$ which contains the origin. Assume that $\sum_{i=1}^{n}\left|\varphi_{i}^{(1)}\right|^{2}$ are strictly pluri- 
subharmonic at the origin. Then there exists a neighborhood $U$ of 0 in D such that the function $\Psi=|f|^{2 k} \sum_{i=1}^{n}\left|\varphi_{i}^{(1)}+f^{\nu} \varphi_{i}^{(2)}\right|^{2}$ is plurisubharmonic (resp. strictly plurisubharmonic) on $U$ (resp. on $U-\{f=0\}$ ) if we take $\nu$ sufficiently large, where $k$ is a positive integer.

Proof. First in general, let $g_{1}, \ldots, g_{t}$ be holomorphic functions defined at the origin of $C^{l}$, and set $G=\sum_{i=1}^{t}\left|g_{i}\right|^{2}$. Then $G$ is obviously plurisubharmonic in a certain neighborhood of the origin. Let $H(G)_{0}$ be the hessian of $G$ with respect to the coordinate system $\left(z_{i}\right)$ at the origin. Then it is easy to see that the number $s$ of positive eigenvalues of $H(G)_{0}$ equals to the dimension $t$ of the natural image of the set $\left\{g_{i}-g_{i}(0)\right\}$ in $m / m^{2}$, where $m$ is the maximal ideal at the origin. In fact note that $H(G)_{0}=H\left(G^{\prime}\right)_{0}$, where $G^{\prime}=\sum_{i=1}^{t}\left|g_{i}^{\prime}\right|^{2}$ and $g_{i}^{\prime}=g_{i} \bmod m^{2}$. In particular for any nonvanishing holomorphic function $h, h G$ has the same number of positive eigenvalues as that of $G$.

Now our function $\Psi$ can be expanded in the form;

$$
\Psi=|f|^{2 k} \sum_{i=1}^{n}\left|\varphi_{i}^{(1)}\right|^{2}+|f|^{2 k}\left\{f^{\nu} \varphi^{\prime \prime}+\bar{f}^{\nu} \bar{\varphi}^{\prime \prime}+|f|^{2 \nu} \varphi^{\prime \prime \prime}\right\},
$$

where $\varphi^{\prime \prime}$ and $\varphi^{\prime \prime \prime}$ are $C^{\omega}$ functions, and $\bar{\varphi}^{\prime \prime}$ denotes the complex conjugate of $\varphi^{\prime \prime}$. Here the first term is clearly plurisubharmonic on $D$ and strictly plurisubharmonic on $U-\{f=0\}$ for some neighborhood $U$ of the origin by the above remark. To get the same conclusion about $\Psi$, recall from the elementary matrix theory that an hermitian matrix is positive definite (resp. positive semidefinite) if and only if all its principal minors are positive (resp. non-negative). Now define a map $\mu: D \subset \mathbb{G}^{l+1}\left(w_{1}, \ldots\right.$, $\left.w_{l+1}\right)$ by $\left(z_{1}, \ldots, z_{l}\right) \rightarrow\left(z_{1}, \ldots, z_{l}, f(z)\right)$, and let $D^{\prime}$ be the biholomorphic image of $D$ by $\mu$. Let $A$ be the zero of $f$ and $\mu(A)=A^{\prime}$. Then $A^{\prime}$ is defind on $D^{\prime}$ by the equation $w_{l+1} \mid D^{\prime}=0$. Further let $\varphi_{i}^{(1) \prime}\left(\operatorname{resp} . \varphi_{i}^{(2) \prime}\right)$ be the holomorphic (resp. $C^{\infty}$ ) extension of $\varphi_{i}^{(1)} \cdot \mu^{-1}\left(\operatorname{resp} . \varphi_{i}^{(2)} \cdot \mu^{-1}\right)$ onto some neighborhood $U^{\prime}$ of the origin of $C^{l+1}$. But since $D^{\prime}$ is nonsingular, we can take $\varphi_{i}^{(1) \prime}$ such that $\sum_{i=1}^{n}\left|\varphi_{i}^{(1)^{\prime}}\right|^{2}$ is strictly plurisubharmonic on $U^{\prime}$. Now put $\Psi^{\prime}=\left|w_{l+1}\right|^{2 k} \sum_{i=1}^{n}\left|\varphi_{i}^{(1) \prime}+w_{l+1}^{\nu} \varphi_{i}^{(2)^{\prime}}\right|^{2}$. This is clearly the extension of $\Psi \cdot \mu^{-1}$ onto $U^{\prime}$. It suffices to prove that this $\Psi^{\prime}$ is pluri- 
subharmonic (resp. strictly plurisubharmonic) on a small neighborhood $U^{\prime \prime}$ of 0 (resp. on $U^{\prime \prime}-A^{\prime}$ ). Let $\Delta$ be any principal minor of the hessian of $\Psi_{(1)}^{\prime}=\left|w_{l+1}\right|^{2 k} \sum_{i=1}^{n}\left|\varphi_{i}^{(1) \prime}\right|^{2}$ and $\Delta^{\prime}$ be that of $\Psi^{\prime}-\Psi_{(1)}^{\prime}$ corresponding to $\Delta$. We see $\Delta>0$ on $U^{\prime}-A^{\prime}$ by the above remark. We show that $\Delta \geqq \Delta^{\prime}$ if $\left|w_{l+1}\right|$ is sufficiently small and strict inequality holds if $w_{l+1} \neq 0$. In fact this follows from the Lojasiewicz inequality [20], but here we prove this, by using the real monoidal transformation according to Hironaka. Let $w_{l+1}=w_{1}+\sqrt{-1} w_{2}$, where $w_{1}$ and $w_{2}$ are the real and imaginary parts of $w_{l+1}$ respectively. And let $\Delta=\sum a_{i_{1} i_{2}} w_{1}^{i_{1}} w_{2}^{i_{2}}$ (resp. $\Delta^{\prime}=$ $\sum a_{i_{1} i_{2}}^{\prime} w_{1}^{i_{1}} w_{2}^{i_{2}}$ ) be the taylor series expansion (resp. the expansion to sufficiently high order) with respect to $w_{1}$ and $w_{2}$. Define a real analytic submanifold $M$ of $\mathbb{C}^{n+1} \times \mathbb{P}^{1}(\mathbb{R})$ by the equation $\eta_{1} w_{2}=\eta_{2} w_{1}$, where $\mathbb{P}^{1}(\mathbb{R})$ is the real projective line and $\left(\eta_{0}: \eta_{1}\right)$ is the real homogeneous coordinate of $\mathbb{P}^{1}(\mathbb{R})$. Let $\rho$ be induced by the projection to the first factor and $B=\rho^{-1}\left(w_{l+1}=0\right) . \quad \mathbb{C}^{l+1} \times \mathbb{P}^{1}(\mathbb{R})$ is covered by two open subsets $W_{i}$ $=\left\{\eta_{i} \neq 0\right\}, W_{i} \cong C^{l+1} \times \mathbb{R}\left(\eta_{j} / \eta_{i}\right) \quad j \neq i$. Set $\left.M_{i}=W_{i}\right\rceil M$. Then $M_{i} \cong C^{l}$ $\times \mathbb{R}^{2}\left(w_{i}, \eta_{j} / \eta_{i}\right) j \neq i$ and $B$ is a real hypersurface defined by $w_{i}=0$ in $M_{i}$. Here the isomorphisms are all real analytic. Now set $\tilde{D}=\rho^{-1}(D)$ and $\rho \mid D=\rho_{D}$. Take an arbitrary point $P$ on $\rho^{-1}(0)$. We may assume that $P \subseteq M_{1}$. Then $\rho^{*}(\Delta)$ and $\rho^{*}\left(\Delta^{\prime}\right)$ are written in the form $\rho^{*}(\Delta)=$ $w_{1}^{a} \tilde{\Delta}_{1}$ and $\rho^{*}\left(\Delta^{\prime}\right)=w_{1}^{b} \Delta_{2}$ respectively, where $\tilde{\Delta}_{1}(P)>0$ and $\tilde{\Delta}_{2} \mid B \not \equiv 0$ because $\rho^{*}\left(\Delta^{\prime}\right)$ does not vanish outside $B$. But the above exponent $b$ can be made arbitrarily large if we take $\nu$ large enough. From this and the properness of the map $\rho$ we infer readily that if we take $\nu$ sufficiently large, then on a suitable neighborhood $\tilde{U}$ of $\rho^{-1}(0)$ in $M$ we have $\rho^{*}(\Delta) \geqq \rho^{*}\left(\Delta^{\prime}\right)$ and the strict inequality holds on $\tilde{U}-B \cap \tilde{U}$. But this in turn gives the desired inequalities on a suitable neighborhood $U_{1}$ of the origin of $\mathbb{C}^{l+1}$. Hence we see that $\Delta \geqq \Delta^{\prime}$ on $U_{1}$ and $\Delta>\Delta^{\prime}$ on $U_{1}-$ $U_{1} \cap A^{\prime}$. Since $\Delta$ was arbitrary, the lemma holds for sufficiently large $\nu$.

Q.E.D.

Remark. For the assertion i) of Lemma 4, the fact that $A$ is an effective Cartier divisor is irrelevant. $A$ may be any weakly l-complete subspace. $A$ similar problem was considered in [8, Prop. 8.1] when $A$ 
is projective.

Proof of Lenma 5. Let $f_{1} \ldots, f_{N}$ be elements of $H^{0}\left(A, \mathcal{O}_{A}\right)$ which defines a proper morphism $F: A \rightarrow \mathbb{G}^{N}$ of $A$ into a complex $n$-space $\mathbb{C}^{N}$ such that $A_{d}=\left\{x \in A ; \sum_{i=1}^{N}\left|f_{i}(x)\right|^{2}<d\right\}$ for any $d \in \mathbb{R}$. Now fix an arbitrary $c \boxminus \mathbb{R}^{+}$and take $d \in \mathbb{R}$ such that $d>2 c$. Then as in the proof of the theorem there exists a neighborhood $U_{d}$ of $A_{d}$ in $X$ on which we can find holomorphic functions $\tilde{f}_{1}, \ldots, \tilde{f}_{N}$ such that $\left.\tilde{f}_{i}\right|_{A_{d}}=\left.f_{i}\right|_{A_{d}}$. On the other hand, by Lemma 4 , there exists a neighborhood $V$ of $A_{d}$ in $X$ on which we can find a plurisubharmonic function $\Psi_{1}$ which is strictly psh. on $V-V \cap A_{d}$. Moreover by the proof of the lemma we may assume that $\Psi_{1}(x)=0, x \in V$, if and only if $x \in A$. Now put $W=V \cap U_{d}$ and $\Psi_{2}=\sum_{i=1}^{n}\left|\tilde{f}_{i}\right|^{2}$. We shall show that for any $d^{\prime}<d$ the subset $W_{\varepsilon, d^{\prime}}$ of $W$ defined by $W_{\varepsilon, d^{\prime}}=\{x \in W$; $\Psi_{1}<\varepsilon$ and $\left.\Psi_{2}<d^{\prime}\right\}$ is relatively compact in $W$, if we take $\varepsilon$ sufficiently small. Assume the contrary. Then there exists a sequence $\left\{\varepsilon_{n}\right\}_{n=1,2} \ldots$ of positive reals which tends to 0 and there associated for each $\varepsilon_{n}$ a sequence $\left\{x_{\alpha}^{(n)}\right\}_{\alpha=1,2} \ldots$ of points of $W_{\varepsilon_{n}}$ such that $\Psi_{2}\left(x_{\alpha}^{(n)}\right)<d^{\prime}$ and $\Psi_{1}\left(x_{\alpha}^{(n)}\right)<\varepsilon_{n}$ for every $\alpha$ and that $x_{\alpha}^{(n)}$ tends to the boundary of $W$ in $X$ if $a \rightarrow \infty$. Take the diagonal sequence $\left\{x_{n}^{(n)}\right\}$. Since we may assume that $W$ is relatively compact in $X$, we can choose a subsequence from $\left\{x_{n}^{(n)}\right\}$ which converges to a point $x_{0}$ on $\partial W$, the boundary of $W$ in $X$. Then by the choice of $\left\{x_{n}^{(n)}\right\}, \Psi_{1}\left(x_{0}\right)=0$ and hence $x_{0} \in A$. Moreover $\Psi_{2}\left(x_{0}\right) \leqq d^{\prime}$. Thus $x_{0}$ is the interior point of $W$, contradicting the assumption.

Now let $\Psi_{1}^{\prime}=2 c / \varepsilon \quad \Psi_{1}$ and $\Psi=\Psi_{1}^{\prime}+\Psi_{2}$, where $\varepsilon$ is chosen for $d^{\prime}=$ 2c. Then the subset $W_{c}=\{\Psi(x)<c\}$ of $W$ is relatively compact and $W_{c} \cap A=A_{c}$. From this we see readily that $W_{r}$ is weakly 1-complete with an exhaustion function $\Psi$ (with upper bound $c$ ). Further if we take $\varepsilon$ small, then $W_{c}$ is arbitrarily small. This completes the proof of the Lemma.

We can also prove the Theorem using Lemmas 4 and 5, by a modification of the method of Grauert [6]. In fact we can prove

Lemma 7. Suppose $X$ is a weakly 1-complete complex space with an exhaustion function $\Psi$, and $A$ is an effective Cartier divisor on $X$. Let $L=[A]$ be the corresponding line bundle. Now assume that $L^{*}$ is 
positive on $X$, and let $x_{0}$ be a point on $\partial X_{c}$ for some $\in \in \mathbb{R}$ such that $\Psi$ is strictly plurisubharmonic at $x_{0}$ and $x_{0} \neq A$. Then there exist a holomorphic function $g$ on $X_{c}$ such that $\lim _{\substack{x_{n \rightarrow x_{0}} \\ x_{n \in X}}} g\left(x_{n}\right)=\infty$.

Proof. By [7, Satz 1.4], there exist a neighborhood $U$ of $x_{0}$ in $X$ and an effective cartier divisor $B^{\prime}$ of $U$ which is stein, and such that $B^{\prime}$ $\cap X_{c}=\left\{x_{0}\right\}$. Take $c^{\prime}>c$ sufficiently near to $c$ so that $B=B^{\prime} \cap X_{c^{\prime}}$ is the analytic subset of $X_{c^{\prime}}$, and that $A \cap B=\phi$. Put $F=[B]$ the corresponding line bundle and consider the exact sequence

$$
0 \longrightarrow \mathcal{O}\left(L^{* \otimes m}\right) \longrightarrow \mathcal{O}\left(L^{* \otimes n} \otimes F\right) \longrightarrow \mathcal{O}_{B}(F) \longrightarrow 0
$$

From this, we obtain a cohomology exact sequence

$$
\begin{aligned}
\longrightarrow H^{0}\left(X_{c^{\prime}}, \mathcal{O}\left(L^{* \otimes^{m}} \otimes F\right)\right) \stackrel{\rho}{\longrightarrow} & H^{0}\left(B, \mathcal{O}_{B}(F)\right) \\
& \longrightarrow H^{1}\left(X_{c^{\prime}}, \mathcal{O}\left(L^{* \otimes^{m}}\right)\right) \longrightarrow .
\end{aligned}
$$

If we take $m$ sufficiently large, then by the vanishing theorem $N^{\prime}$ we have $H^{1}\left(X_{c^{\prime}}, \mathcal{O}\left(L^{* \otimes^{m}}\right)\right)=0$, and hence the map $\rho$ is surjective. Since $B$ is stein, we can find an element $s \in H^{0}\left(B, \mathcal{O}_{B}(F)\right)$ such that $s_{x_{0}} \neq 0$. Let $\tilde{s}$ be an element of $H^{0}\left(X_{c^{\prime}}, \mathcal{O}\left(L^{* \otimes m} \otimes F\right)\right)$ which lifts $s$. By virtue of the natural isomorphism $\mathcal{O}\left(L^{* \otimes m}\right)=\mathcal{G}_{A}^{m}$, $\tilde{s}$ can be regarded as an element $\tilde{s}^{\prime}$ of $H^{0}\left(X_{c^{\prime}}, \mathcal{O}(F) \otimes \mathcal{G}_{A}^{m}\right)$. Then clearly, this $\tilde{s}^{\prime}$ gives a meromorphic function $g^{\prime}$ on $X_{c^{\prime}}$ which has a pole on $B$ and is holomorphic outside $B$. But since $B \cap X_{c}=\left\{x_{0}\right\}, g=g^{\prime} \mid X_{c}$ is the desired function. Q.E.D. As a Corollary we have

Corollary. Suppose $X$ and $A$ are as in Theorem 1, except that we replace the condition 1) by the following weaker condition $1^{\prime}$ ).

1') $X$ is weakly 1-complete.

Then for any $c \in \mathbb{R}$ there exists a relatively compact neighborhood $W_{c}$ of $A_{c}$ in $X$ such that for every point $x_{0} \in \partial W_{c}-\partial V_{c} \cap A$ there exists an element $g_{x_{0}} \in H^{0}\left(W_{c}, O_{W_{c}}\right)$ such that $\lim _{\substack{x \rightarrow x_{0} \\ x \in W_{c}}} g_{x_{0}}(x)=\infty$.

If we further assume that $A$ is holomorphically convex, then we can find also for the point $x_{1}$ on $\partial W_{c} \sqcap A$ an element $g_{x_{1}}$ with the similar property. Thus under the assumptions of Theorem 1, we can find for any $c \in \boldsymbol{R}$, a holomorphically convex neighborhood $U_{c}$ of $A_{c}$ in $X$ such that 
$U_{c} \cap A=A_{c}$, and that $\exists \Psi$, a plurisubharmonic function on $U_{c}$ which is strictly plurisubharmonic on $U_{c}-A_{c}$. Let $f_{c}: U_{c} \rightarrow \bar{U}_{c}$ be the Remmert quotient of $U_{c}$. Then $f_{c}$ is isomorphic outside $A_{c}$. In fact, if $\operatorname{dim}$ $f_{c}^{-1}(y)>0$ for some $y \in \bar{U}_{0}-f_{0}\left(A_{c}\right)$, then $\Psi$ must be constant on $f_{c}^{-1}$ $(y)$, and this contradicts with the fact that $\Psi$ is strictly plurisubharmonic on $U_{c}-A_{c}$ (c.f. [26, Lemma 3.2]). But then since the fibre of $f_{c}$ is connected and $f_{c *} \mathcal{O}_{U c}=\mathcal{O}_{U c}, f_{c}$ is isomorphic outside $A_{c}$. This will prove the theorem.

An example of the pair $X$ and $A$ satisfying the conditions 1$\left.)^{\prime}, 2\right), 3$ ) but not 1), was constructed in [5].

In the following remark, we shall discuss the case that $A \subset_{-} X$ is not a cartier divisor in Theorem 1 .

Remark 1 . Suppose $A$ is a subspace of a complex space $X$, which is holomorphically convex. Perform a monoidal transformation $\sigma: \tilde{X} \rightarrow X$ with center $A$ and put $\tilde{A}=\sigma^{-1}(A)$. Then $\tilde{A}$ is an effective cartier divisor on $\tilde{X}$. In this situation if the pair $(\tilde{X}, \tilde{A})$ satisfies the conditions 1)-3) of Theorem 1 and if $X$ is normal, then the conclusion of the theorem holds also for the pair $(X, A)$, namely, for any $c \in \mathbb{R}$ there exists a neighborhood $U_{c}$ of $A_{c}$ in $X$ which admits a proper morphism $\iota: U_{c} \rightarrow \mathbb{C}^{m}$ into some number space $C^{m}$, which is isomorphic outside $A_{c}$. In fact, first we apply the Theorem to the pair $(\tilde{X}, \tilde{A})$ to obtain for any $c \in \mathbb{R}$ a neighborhood $\tilde{U}_{c}$ of $\tilde{A}_{c}$ in $\tilde{X}$ and a finite number of elements $f_{1}, \ldots, f_{n} \in H^{0}\left(\tilde{U}_{c}, O_{\tilde{U} c}\right)$ such that the map $F: \tilde{U}_{c} \rightarrow \mathbb{C}^{n}$ defined by them is proper and isomorphic outside $\tilde{A}_{c}$. Here we may take $\tilde{A}_{c}$ and $A_{c}$ so as to $\tilde{A}_{c}=\sigma^{-1}\left(A_{c}\right)$. Now the normality of $X$ implies $\sigma_{*}\left(\mathcal{O}_{\tilde{X}}\right)=\mathcal{O}_{X}$ and hence the natural inclusion $H^{0}\left(U_{c}, \mathcal{O}_{X}\right) \subset H^{0}\left(\tilde{U}_{c}, \mathcal{O}_{\tilde{X}}\right), U_{c}=\sigma\left(\tilde{U}_{c}\right)$, is an isomorphism. Thus we may consider $f_{i}$ as elements of $H^{0}\left(U_{c}, \mathcal{O}_{X}\right)$, (which will then be denoted by $\bar{f}_{i}$, and the map $F$ factors through $\sigma \mid \tilde{U}_{c}: \tilde{U}_{c} \rightarrow U_{c}$ and $\bar{F}: U_{c} \rightarrow \mathbb{C}^{n}$, where $\bar{F}$ is defined by $\bar{f}_{i}$. These $U_{c}$ and $\bar{F}$ are the desired ones.

But unfortunately I could find no nice conditions for the pair $(\tilde{X}$, $\tilde{A})$ to satisfy the requirements of the Theorem, for instance, in terms of the normal cone $C_{X, A}=\operatorname{Specan}\left(\bigoplus_{m=0}^{\infty} \mathcal{G}_{A}^{m} / \mathcal{G}_{A}^{m+1}\right)$ of $A$ in $X[16$, II]. (But c.f. $\S 3$ ). Here we only note the following fact in this connection; there exists a 
proper morphism $\sigma_{0}: C_{\tilde{X}, \tilde{A}}=N_{\tilde{A} / \tilde{X}}=[\tilde{A}]_{\tilde{A}} \rightarrow C_{X, A}$, which is isomorphic to the monoidal transformation with center the vertex $(\cong A)$ of the cone $C_{X, A}$ (c.f. [9, I (8.7.7)]). Let $i: A \subseteq C_{X, A}$ be the inclusion of $A$ as the vertex of $C_{X, A}$ and $p: C_{X, A} \rightarrow A$ the natural projection. Consider for example the following condition $\left(^{*}\right)$ for the normal cone $C_{X, A}$;

${ }^{*}$ ) there exists a strictly plurisubharmonic function $\Psi$ on $C_{X, A}-i(A)$ such that $p \mid C_{X, A}^{d}: C_{X, A}^{d} \rightarrow A$ is proper, where $C_{X, A}^{d}=\left\{P \in C_{X, A} ; \Psi(P) \leqq d\right\}$. Then by the above remark, $C_{X, A}$ satisfies the condition $\left(^{*}\right)$ if and only if $C_{\tilde{X}, \tilde{A}}$ satisfies the corresponding one, (if $X$ is normal). If $A$ is compact, then it is well-known that, $\left(^{*}\right)$ for $C_{\tilde{X}}, \tilde{A}=N_{\tilde{A} / \tilde{X}}$ is equivalent to the fact that the line bundle $N_{\tilde{A} / \tilde{X}}^{*}$ is positive in the sense of Definition 1 [7], [9], and hence in particular $\left({ }^{*}\right)$ for $C_{X, A}$ implies the condition (2) of Theorem 1. Thus we get the following result corresponding to Satz 3.8 of [7]; Suppose $X$ and $A$ are as above. If $A$ is compact and $C_{X, A}$ satisfies the condition (*), then $A$ is exceptional in $X$ in the sense of [7]. But I don't know whether the analogous fact holds or not, if we assume only that $A$ is holomorphically convex.

\section{§3. Globalization}

Now we come back to the original problem of finding the sufficient condition for the blowing-down as stated in the introduction.

3.1. Suppose $X, A$, and $f: A \rightarrow A^{\prime}$ are as in the introduction. First we introduce a coherent analytic subsheaf $\mathcal{S}=\mathcal{S}_{X, A, f}$ of $\mathcal{O}_{X}$ as follows;

$$
\begin{aligned}
\mathcal{S}_{x}: & =\mathcal{O}_{X, x} \text { if } x \notin A, \text { and } \\
& =(r)^{-1}\left(f^{-1}\left(\mathcal{O}_{A^{\prime}, y}\right)\right) \text {, with } y=f(x), \text { if } \quad x \in A,
\end{aligned}
$$

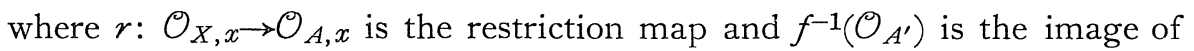
$f^{*}\left(\mathcal{O}_{A^{\prime}}\right)$ in $\mathcal{O}_{A}$. By definition, we have the exact sequence of $\mathcal{O}_{X}$-modules

$$
o \longrightarrow \mathcal{S} \longrightarrow \mathcal{O}_{X} \longrightarrow \mathcal{O}_{A} \mid f^{-1}\left(\mathcal{O}_{A^{\prime}}\right) \longrightarrow 0 .
$$

Thus $\mathcal{S}$ is coherent. The motivation for this is

Proposition 1. Assume that a blowing-down $\left(X^{\prime}, f^{\prime}\right)$ of $X$ along $f$ exists. Then there exists a blowing-down $(\bar{X}, \bar{f})$ of $X$ along $f$ satisfying 
the following equivalent conditions;

1) For any blowing down $\left(X^{\prime \prime}, f^{\prime \prime}\right)$ of $X$ along $f$, there exists a unique morphism $h: \bar{X} \rightarrow X^{\prime \prime}$ such that $h \cdot \bar{f}=f^{\prime \prime}$.

2) The relation $f_{*}\left(S_{X, A, f}\right)=\mathcal{O}_{\bar{X}}$ holds.

Hence in particular $(\bar{X}, \bar{f})$ is unique except for isomorphisms. We call this $(\bar{X}, \bar{f})$, the universal (or Remmert) blowing down of $X$ along $f$. But we do not prove this proposition here, because the method of the proof is essentially contained in the proof of the next Theorem. Now we show the following theorem by patching together the local blowing downs obtained in Theorem ].

Theorem 2. Suppose $X$ is a complex space, $A$ an effective cartier divisor on $X$ and $f: A \rightarrow A^{\prime}$ a proper surjective morphism of $A$ onto another complex space $A^{\prime}$. Let $L=[A]$ be the corresponding line bundle and $L_{A}=\left.L\right|_{A}$, the restriction on $A$. Now assume the following conditions;

1) $L_{A}^{*}$ is $f$-ample [10].

2) $R^{1} f_{*}\left(L_{A}^{* \otimes \mu}\right)=0$ for every $\mu>0$.

Then there exists a blowing down $\left(X^{\prime}, f^{\prime}\right)$ of $X$ along $f$ such that $f_{*}^{\prime} \mathcal{S}_{X, A, f}$ $=O_{X^{\prime}}$.

Remark. We say that a line bundle $F$ on $A$ is $f$-positive, if for any point $a^{\prime} \in A^{\prime}$ there exists a neighborhood $U_{a^{\prime}}$ of $a^{\prime}$ in $A^{\prime}$ such that $F$ is positive on $f^{-1}\left(U_{a^{\prime}}\right)$ in the sense of Definition 1 . Then as follows from Lemma $3,(1)$ is equivalent to saying that $L_{A}^{*}$ is $f$-positive.

Proof. Let $a^{\prime} \in A^{\prime}$ be any point, $U^{\prime}=U^{\prime}{ }^{\prime}$ a stein neighborhood of $a^{\prime}$ in $A^{\prime}$, and $U=f^{-1}\left(U^{\prime}\right)$. But in the following, we replace $U^{\prime}$ by a smaller neighborhood, if necessary, without a particular mention of it. Taking $U^{\prime}$ sufficiently small, we may assume that $L_{A}^{*}$ is positive on $U$ by virtue of the above Remark. On the other hand, by the Leray spectral sequence for the map $\left.f\right|_{U}: U \rightarrow U^{\prime}$ and by the condition 2), we have $H^{1}\left(U, \mathcal{O}\left(L_{A}^{* \mu}\right)\right)=0$ for any $\mu>0$. Now let $V$ be the open neighborhood of $U$ in $X$ such that $V \cap A=U$. Then the pair of the complex space $V$ and the subspace $U$ of $V$ satisfies all the conditions of Theorem 1, since 
$U$ clearly is holomorphically convex. Hence by (the proof of) that theorem we get the following situation; there exists a neighborhood $W$ of $U$ in $V$, and a finite number of elements $f_{1}, \ldots, f_{n}$ of $H^{0}\left(W, \mathcal{O}_{W}\right)$ such that 1) they define a proper holomorphic map $\tau: W \rightarrow D_{\varepsilon}=\left\{\left(z_{i}\right) \in C^{n}\right.$; $\left.\left.{ }_{\mid z i} \mid<\varepsilon\right\} \quad 2\right) \tau \mid W-U$ is isomorphic, and 3) $\left.\tau\right|_{U}$ coincides with $f_{U}=\left.f\right|_{U}$. Thus we have the following commutative diagram which means the blowing down of $W$ along $f$;

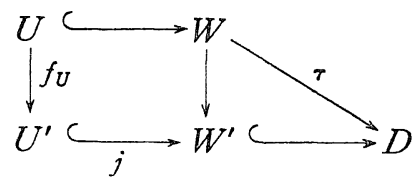

where $W^{\prime}$ is the analytic subset of $D$ which is the image of $W$ and $j$ is the closed imbedding, the existence of which is assured by 3) above.

Note that by 3 ) above, $f_{i}$ actually are the elements of $H^{0}(W, \mathcal{S}), \mathcal{S}$ being defined by (1). Now by the Grauert direct image theorem, the sheaf $\tau_{*}(\mathcal{S})$ is a coherent $\mathcal{O}_{D}$-module, and then by Theorem $A$, (if we replace $U$ by a relatively compact subdomain,) we can find a finite number of elements $s_{1}, \ldots, s_{k}$ of $H^{0}(W, \mathcal{S})$ in such a way that $s_{i}(a)=0$ if $f(a)=$ $a^{\prime}$, and $\tau_{*}\left(s_{i}\right)$ generate $\tau_{*}(\mathcal{S})$ at every point of $D$. Then if we take $\varepsilon^{\prime}$ sufficiently small, a map $\tau_{1}$ defined by

$$
\tau_{1}=\tau \times\left(s_{1}, \ldots, s_{k}\right): W \longrightarrow D_{1}=D \times D^{\prime}, D^{\prime}=\left\{\left(z_{i}\right) \in C^{k} ;\left|z_{i}\right|<\varepsilon^{\prime}\right\},
$$

is proper (replacing $U$ by a smaller one). Further, by the definition of $\mathcal{S}$ we still have $\left.\tau_{1}\right|_{U}=f$, and have the inclusion $j_{1}: U \subseteq W_{1}$, where $W_{1}$ is the image of $W$ by the map $\tau_{1}$. We have the commutative diagram:

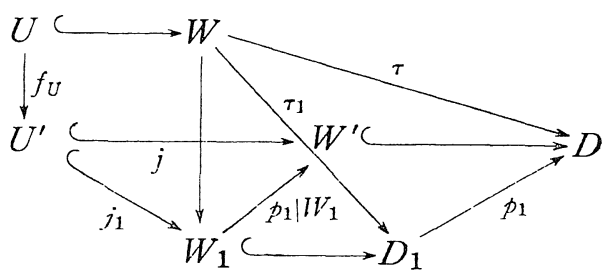

where $p_{1}$ is the projection to the first factor, and it is easily seen that $\left.p_{1}\right|_{W_{1}}$ induces a homeomorphism of the underlying topological space of $W^{\prime}$ and $W_{1}^{\prime}$. From this, we conclude readily that $\tau_{1 *}(\mathcal{S})$ is (coherent and) 
generated by $\tau_{1 *}\left(s_{k}\right)$ at each point of $D_{1}$. This means in particular that every elements $s$ of $H^{0}(W, \mathcal{S})$ can be written as a pull back of the convergent power series of $\left(z_{i}\right)$ defined on $D_{1}$. Hence we have $\tau_{1 *}(\mathcal{S})=\mathcal{O}_{W_{1}}$. For these arguments see [27]. Therefore, we may assume from the beginning that $\tau_{*}(\mathcal{S})=\mathcal{O}_{W}$ in (2), replacing $\tau$ by $\tau_{1}$. Now take and fix for every point $a^{\prime} \in A^{\prime}$ a diagram as in (2). We put a suffix $a^{\prime}$ for the corresponding letters, e.g. $W^{\prime} a^{\prime}, \tau_{a^{\prime}}$, etc.

Suppose $U_{a_{1}^{\prime}} \cap U_{a_{2}^{\prime}} \neq \phi$ for $a_{1}^{\prime} \neq a_{2}^{\prime}$ and define a map $\tau_{12}: \tau_{x_{1}^{\prime}}\left(I V_{a_{1}^{\prime}}^{\prime} \cap W_{a_{2}^{\prime}}^{\prime}\right)$ $\rightarrow \tau_{a_{2}^{\prime}}\left(W_{a_{1}^{\prime}}^{\prime} \cap W_{a_{2}}^{\prime}\right)$ by $\tau_{12}=\tau_{a_{2}} \tau_{a_{1}}^{-1}$. Put $W_{1}^{\prime}=W_{a_{i}}^{\prime}, i=1,2, W_{12}^{\prime},=W_{1}^{\prime} \cap$ $W_{2}^{\prime}$ and $\tau_{i}=\tau_{a_{i}^{\prime}}$. Then since the map $\tau_{1}$ and $\tau_{2}$ give topologically equivalent fiberings on $W_{12}^{\prime}, \tau_{12}$ is well-defined and induces a homeomorphism of the underlying topological spaces. Further, since $\mathcal{O}_{W_{2}^{\prime} b^{\prime}}=\tau_{2 *}(\mathcal{S})_{b^{\prime}}=$ $H^{0}\left(\tau_{2}^{-1}\left(b^{\prime}\right), \mathcal{S}\right)=H^{0}\left(\tau_{1}^{-1}(b), \mathcal{S}\right)=\tau_{1 *}(\mathcal{S})_{b}=\mathcal{O}_{W_{1}^{\prime}, b}$ if $\tau_{12}(b)=b^{\prime}$, we have natural isomorphisms $\tau_{12, b b^{\prime}}^{*}: \mathcal{O}_{W_{2}^{\prime}, b^{\prime}} \cong \mathcal{O}_{W_{1}^{\prime}, b}$. Let $\tau_{12}^{*}=\left\{\tau_{12, b, b^{\prime}}^{*}\right\}$. Then it is easy to see that the morphism of ringed spaces $\left(\tau, \tau^{*}\right):\left(\tau_{1}\left(W_{12}^{\prime}\right), \mathcal{O}_{W_{1}^{\prime}}\right)$ $\rightarrow\left(\tau_{2}\left(W_{12}^{\prime}\right), \mathcal{O}_{W_{2}^{\prime}}\right)$ gives an isomorphism of the analytic spaces. Moreover since if $W_{a_{1}^{\prime}} \cap W_{a_{2}^{\prime}} \cap W_{a_{3}^{\prime}} \neq \phi$, then we have $\tau_{23}{ }^{\circ} \tau_{12}=\tau_{13}$, we conclude that $W^{\prime}=\bigcup_{a^{\prime} \in A^{\prime}} W_{a}^{\prime}$, with the identification by $\tau_{a^{\prime} a^{\prime \prime}}$, defines a well-defined complex space and $\tau^{\prime}=\bigcup_{a^{\prime} \in A^{\prime}} \tau_{a^{\prime}}: W=\bigcup_{a^{\prime} \in A^{\prime}} W_{a^{\prime}} \rightarrow W^{\gamma^{\prime}}$ a morphism of complex spaces. We have naturally an inclusion $A^{\prime} \subseteq W^{\prime}$. Finally we set $X=(X$ - A) $\cup W^{\prime}$, where $X-A$ and $W^{\prime}-A^{\prime}$ are identified by the isomorphisms $\tau_{a^{\prime}}: W_{a^{\prime}}-W_{a^{\prime}} \Gamma_{1} A \cong W_{a^{\prime}}^{\prime}-W_{a^{\prime}}^{\prime} \cap A^{\prime}$, and set $f=\tau^{\prime}$ on $W$ and $=$ identity on $X-A$. Then it is immediately seen that $f: X \rightarrow Y^{\prime}$ is welldefined and gives the blowing down of $X$ along $f$. The relation $f_{*}^{\prime} \mathcal{S}_{X, A, f}=\mathcal{O}_{X^{\prime}}$ follows from construction.

Q.E.D.

Colollary. Suppose $X$ is a reduced complex space, $A \subseteq X$ a subspace which is nowhere dense in $X$, and $f: A \rightarrow A^{\prime}$ a proper surjective morphism, where $A^{\prime}$ is another complex space. Let $C_{X, A}$ be the normal cone of $A$ in $X$ (see Remark 1), and $i: A \subseteq C_{X, A}$ the natural inclusion as a vertex. Now assume that

1) $C_{X, A}$ can be blown down along $f$, and

2) $R^{1} f_{*}\left(\mathcal{I}^{\mu} / \mathcal{G}^{\mu+1}\right)=0$ for every $\mu>0$, where $\mathcal{I}$ is the ideal sheaf of $A$. 
Then, there exists a blowing down $\left(X^{\prime}, f^{\prime}\right)$ of $X$ along $f$ with the property that $f_{*}^{\prime} \mathcal{S}=O_{X^{\prime}}$.

Proof. Let $\sigma: \tilde{X} \rightarrow X$ be the blowing up with center $A$ and $\tilde{A}=$ $\sigma^{-1}(A)$. Then by Remark 1 we have a bimeromorphic morphism $\sigma_{0}$ : $C_{\tilde{X}, \tilde{A}} \rightarrow C_{X, A}$ which is isomorphic to the blowing up with center $A \complement_{\longrightarrow} C_{X, A}$. Here $C_{\tilde{X}}, \tilde{A}$ now coincides with the normal bundle $N_{\tilde{A} / \tilde{X}}$ of $\tilde{A}$ in $\tilde{X}$, and the inclusion $i: \tilde{A} \subset C_{\tilde{X}}, \tilde{A}$ is as the 0 -section. We set $E=C_{\tilde{X}}, \tilde{A}$. Let $f_{1}=f \cdot \sigma \mid \tilde{A}$, and $h: C_{X, A} \rightarrow C_{X, A}^{\prime}$ the universal blowing down of $C_{X, A}$ with respect to $f$, so that $h_{*} \mathcal{S}_{C_{X, A}, A, f}=\mathcal{O}_{C_{X, A}^{\prime}}$ (c.f. Prop. 1). Then $h_{1}$ $=h \cdot \sigma_{0}$ gives the blowing down of $E$ along $f_{1}$. Let $\tilde{p}: E \rightarrow \tilde{A}$ (resp. $p: C_{X, A}$ $\rightarrow A)$ be the structure morphism. Then, since $(f \cdot p \cdot i)^{-1} \mathcal{O}_{A^{\prime}}=f^{-1} \mathcal{O}_{A^{\prime}},(f \cdot$ p) $-1 \mathcal{O}_{A^{\prime}}$ is contained in the sheaf $\mathcal{S}_{C_{X}, A^{\prime}, A}$. Hence we infer that the (continuous) map $p^{\prime}=f \cdot p \cdot h^{-1}: C_{X, A}^{\prime} \rightarrow A^{\prime}$ is holomorphic. Hence we have the commutative diagram

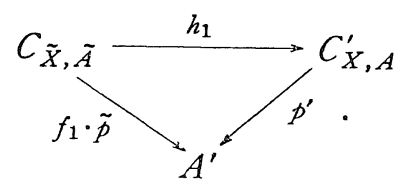

But then, by [17, Satz 5.5], there exists for any point $a^{\prime} \in A^{\prime}$ a neighborhood $U_{0}$ of $a^{\prime}$ in $A^{\prime}$ and an open set $W$ in $\left(f_{1} \cdot p\right)^{-1}\left(U_{0}\right) \subset E$, such that i) $i\left(f^{-1}\left(U_{0}\right)\right) \subset W$, and ii) $\left.p\right|_{W}: W \rightarrow f^{-1}(U)$ is 1-convex (see Def. 1.1 of [17]). Then by [17, Theorem 6.4], the dual $E^{*} \rightarrow \tilde{A}$ is $f_{1}$-ample. Take now a sufficiently small stein open subset $U \subset A$, which is yet to be determined in the sequel. Let $V=f^{-1}(U)$. Then by 2$) H^{1}\left(V, \mathcal{G}^{\mu} / \mathcal{G}^{\mu+1}\right)=0$ and hence

$$
H^{0}\left(V, \mathcal{O}_{X} / \mathcal{G}^{\mu+1}\right) \longrightarrow H^{0}\left(V, \mathcal{O}_{X} / \mathcal{I}^{\mu}\right) \longrightarrow 0
$$

is exact for every $\mu>0$. On the other hand, let $\tilde{V}=f_{1}^{-1}(U)$. We may

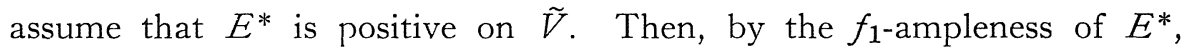
there exists a positive integer $m_{0}>0$ such that $H^{1}\left(\tilde{V}, E^{* \otimes^{m}}\right)=H^{1}\left(\tilde{V}, g^{m}\right)$ $=0$ and hence $H^{0}\left(\tilde{V}, \mathcal{O}_{\tilde{X}} / g^{m+1}\right) \stackrel{a_{m}}{\longrightarrow} H^{0}\left(V, \mathcal{O}_{\tilde{X}} / g^{m}\right)$ is surjective for every $m>m_{0}$, where $g=\sigma^{-1}(\mathcal{G})$ is the ideal sheaf of $\tilde{A}$ in $\tilde{X}$. Now let $g$ be any 
element of $H^{0}\left(\tilde{V}, f_{1}^{-1} \mathcal{O}_{U}\right)=H^{0}\left(V, f^{-1} \mathcal{O}_{U}\right)$. Then by $(3)$ we can extend this to an element $g_{1}$ of $H^{0}\left(V, \mathcal{O}_{X} / g^{m+1}\right)$. Let $g^{\prime} \in H^{0}\left(\tilde{V}, \mathcal{O}_{X} / g^{m+1}\right)$ be the pull-back of $g_{1}$. Then by the surjectivity of $\alpha_{m}$, for every $\nu>$ $m_{0}$ there exists an element $g_{\nu} \in H^{0}\left(\tilde{V}, \mathcal{O}_{\tilde{X}} / g^{\nu+1}\right)$ which extends $g^{\prime}$.

Now define an analytic subsheaf $\mathcal{S}^{\prime}$ of $\mathcal{O}_{\tilde{X}}$ by $\mathcal{S}^{\prime}=\mathcal{S}_{\tilde{X}}, \tilde{A}, f_{1} \cap \sigma^{-1}\left(\mathcal{O}_{X}\right)$ $=\sigma^{-1}\left(\mathcal{S}_{X, A, f}\right)$, where $\mathcal{S}_{\tilde{X}, \tilde{A}, f_{1}}$ and $\mathcal{S}_{X, A, f}$ are defined by (1). Then exactly as in the proof of the Theorem we construct a diagram (2) for which $\tau_{*}\left(\mathcal{S}^{\prime}\right)=\mathcal{O}_{W}$ this time, and then patching together these $W_{a^{\prime}}$ and $\tau_{a^{\prime}}$, defined for each $a^{\prime}$ as in the proof of the theorem, we get the blowing down $\left(X^{\prime}, f_{1}^{\prime}\right)$ of $\tilde{X}$ along $f_{1}$ for which $f_{1 *}^{\prime} \mathcal{S}^{\prime}=\mathcal{O}_{X^{\prime}}$. But then, since $\sigma$ is surjective, we see that the map $f^{\prime}=f_{1}^{\prime} \sigma^{-1}$ is well-defined and continuous as a map of the underlying topological spaces. Moreover, that this $f^{\prime}$ is induced by the morphism of analytic spaces follows easily from $f_{1}^{\prime-1}\left(\mathcal{O}_{X^{\prime}}\right)=\sigma^{-1}\left(\mathcal{S}_{X, A, f}\right)$. Hence $\left(X^{\prime}, f^{\prime}\right)$ is the desired blowing down.

Q.E.D.

Remark 2. Suppose $X, A$, and $f: A \rightarrow A^{\prime}$ are as in the corollary. We assume, however, only the condition 2 ) of the corollary. Let $g$ be the coherent sheaf of ideals of $\mathcal{O}_{X}$ whose support coincides with $A_{\text {red }}$, and which is contained in $\mathcal{G}=\mathscr{S}_{A}$. Let $B$ be the subspace of $X$ corresponding to $\mathcal{G}$. Then by (2), as in the proof of the corollary there exists a proper surjective morphism $f_{B}: B \rightarrow A^{\prime}$ which extends $f$. With this remark in mind, the following three conditions are equivalent.

1) $X$ can be blown down along $f$.

2) There exists coherent ideal sheaf $g^{\prime}$ of $\mathcal{O}_{X}$ such that if $\sigma: \tilde{X} \rightarrow X$ is the blowing up of $X$ with center $g^{\prime}$, then the sheaf $\tilde{y}^{\prime} \otimes \mathcal{O}_{B^{\prime}}$ is $\left(\left.f_{B^{*} \sigma}\right|_{B^{\prime}}\right)$ ample, where $\tilde{g}^{\prime}=f^{-1}\left(g^{\prime}\right)$ and $B^{\prime}$ is the subspace of $\tilde{X}$ defined by $\tilde{g}^{\prime}$.

3) There exists a coherent ideal sheaf $\mathscr{g} \subseteq \mathcal{O}_{X}$ with support $A_{\text {red }}$ and $\mathscr{g} \subseteq \mathcal{G}$ as above with the following property; let $B$ be the subspace of $X$ defined by $g$. Then the normal cone $C_{X, B}$ of $B$ in $X$ can be blown down along $f_{B}: B \rightarrow A^{\prime}$ for some $f_{B}$ as above.

Proof. 1) $\rightarrow 2$ ). Let $f^{\prime}: X \rightarrow X^{\prime}$ be the blowing down of $X$ along $f$. By Chow lemma [12] there exists a coherent sheaf of ideals $\mathcal{G}_{0}$ of $\mathcal{O}_{X^{\prime}}$ with support $A_{\text {red }}^{\prime}=f(A)_{\text {red }}$ such that if $\tilde{f}: \tilde{X} \rightarrow X^{\prime}$ is the blowing up of 
$X^{\prime}$ with center $\mathcal{I}_{O}$, then we have a proper surjective morphism $h: \widetilde{X} \rightarrow X$ such that $\tilde{f}=f^{\prime} \cdot h$. Then by [14, Lemma 4], $h$ is the blowing up of $X$ with center $\mathcal{G}=\left(f^{\prime}\right)^{-1}\left(\mathcal{I}_{0}\right)$. Since $(\tilde{f})^{-1}\left(\mathcal{G}_{0}\right)=h^{-1}(\mathcal{G})$ is $\tilde{f}$-(very) ample (c.f. $\S(b))$, this implies 2).

$2) \rightarrow 3$ ). We put $\mathscr{g}=\mathcal{g}^{\prime}$ and let $B$ be the subspace defined by $\mathscr{g}$ and $\widetilde{B}=\sigma^{-1}(B)$. Then by Remark 1 we have a bimeromorphic morphism $\sigma_{0}: C_{\tilde{X}, \tilde{B}} \rightarrow C_{X, B}$, isomorphic to the blowing up of $C_{X, B}$ with center the vertex $\left(\cong B\right.$ ) of $C_{X, B}$. But since $C_{\tilde{X}, \tilde{B}}$ coincides with normal bundle $N_{\tilde{B} / \tilde{X}}$ of $\tilde{B}$ in $\tilde{X}$ and $\mathcal{O}\left(N_{\tilde{B} / \tilde{X}}^{*}\right)=\tilde{g}_{\tilde{B}} \otimes \mathcal{O}_{\tilde{B}},[\tilde{B}]_{\tilde{B}}=N_{\tilde{B} / \tilde{X}}^{*}$ is $f_{\tilde{B}}\left(\left.\sigma_{0}\right|_{\tilde{B}}\right)$-ample. Moreover because of the structure morphism $e: C_{\tilde{X}}, \tilde{B} \rightarrow \tilde{B}$ and the 0 -section $i: \tilde{B} \rightarrow C_{\tilde{X}} \tilde{B}$ of $e$, the arguments similar to the above Corollary shows that we have a blowing-down $\sigma_{1}: C_{\tilde{X}, \tilde{B}} \rightarrow C_{\tilde{X}, \tilde{B}}^{\prime}$ of $C_{\tilde{X}, \tilde{B}}$ along $\left.f_{B^{*}} \sigma_{0}\right|_{\tilde{B}}: \widetilde{B} \rightarrow A$ such that $\sigma_{1}$ factors through $\sigma_{1}: C_{\tilde{X}, \tilde{B}} \stackrel{\sigma_{11}}{\longrightarrow} C_{X, B} \stackrel{{ }^{\sigma 12}}{\longrightarrow} C^{\prime} \tilde{X}, \tilde{B}$. Then the pair $\left(C_{\tilde{X}, \tilde{B}}^{\prime}, \sigma_{12}\right)$ is seen to be the desired blowing down.

That 3$) \rightarrow 1$ ) is the above Corollary. It may be interesting to give the proof of 1) $\rightarrow 2$ ) not using Chow lemma.

Remark 3. It is easy to generalize the corollary to the following relative situation. Suppose $X, A$ and $f: A \rightarrow A^{\prime}$ are as in the corollary and $S$ a complex space. Assume that $X, A^{\prime}$ are complex spaces over $S$ and $f$ is an $S$-morphism, where $A$ has the natural structure of a complex space over $S$. Then the blowing down $\left(X^{\prime}, f^{\prime}\right)$ obtained in the corollary has the additional property that $X^{\prime}$ is a complex space over $S$ and $f^{\prime}$ is an $S$-morphism. In fact, it is easily seen that $\pi_{X^{\prime}}=\pi_{X}\left(f^{\prime}\right)^{-1}$ is a continuous map, where $\pi_{X}$ is the structure morphism. On the other hand, we have $i^{-1} \pi_{X}^{-1}\left(\mathcal{O}_{S}\right)=\left(\pi_{A^{\prime}} f\right)^{-1}\left(\mathcal{O}_{S}\right)=f^{-1}\left(\mathcal{O}_{A^{\prime}}\right), \pi_{X}^{-1}\left(\mathcal{O}_{S}\right) \subset \mathcal{S}_{X, A, f}$ and hence $f_{*}^{\prime}\left(\pi_{X}^{-1}\right.$ $\left.\left(\mathcal{O}_{S}\right)\right) \leqq f_{*}^{\prime} \mathcal{S}=\mathcal{O}_{X^{\prime}}$. From this we conclude that $\pi_{X}$ is a morphism of complex spaces. In this case we say that $X$ can be blown down along $f$ over $S$.

In [4], it is shown that every small deformation of a monoidal transformation is again a monoidal transformation. As an easy application of Theorem 2 together with Remark 3 above, we show the following Proposition which generalizes this result. 
Proposition 2. Let $S$ be a complex space, $X, A^{\prime}$ complex spaces over $S, A$ an effective cartier divisor on $X$, and $f: A \rightarrow A^{\prime}$ a proper surjective and Aat S-morphism, where $A$ has the natural structure of an S-space by $\left.\pi_{X}\right|_{A}, \pi_{X}$ being the structure morphism of $X$. Set $L=[A]$, the line bundle corresponding to $A$. Let $s_{0} \in S$ be a point and denote by $X_{0}$ (resp. $\left.A_{\mathbf{0}}, A_{\mathbf{0}}^{\prime}\right)$ the fiber over $s_{\mathbf{0}}$ of $X$ (resp. $\left.A, A^{\prime}\right)$. Nore assume the following conditions;

1) the restriction $L_{A_{0}}$ of $L$ onto $A_{0}$ is $f_{0}$-ample, where $f_{0}=\left.f\right|_{A_{0}}$.

2) $R^{1} f_{0 *}\left(\mathcal{O}\left(L_{A 0}^{* \mu}\right)=0\right.$ for every $\mu>0$.

Then, we can find a neighborhood $U^{\prime}$ of $A_{0}^{\prime}$ in $A^{\prime}$ with the property that, if we set $U=f^{-1}\left(U^{\prime}\right)$, then there exists a neighborhood $W$ of $U$ in $X$ with $W \cap A=U$, such that $W$ can be blown down along $\left.f\right|_{U}$ over $S$.

Proof. First we show that for any point $a^{\prime} \in A_{0}^{\prime}$, there exists a neighborhood $U_{a^{\prime}}^{\prime}$ of $a^{\prime}$ in $A^{\prime}$ with the following properties; $\left(^{*}\right)$ if we set $U_{a^{\prime}}=f^{-1}\left(U_{a^{\prime}}^{\prime}\right)$, then 1) $\left.a^{\prime} L\right|_{U_{a^{\prime}}}$ is $\left.f\right|_{U_{a^{\prime}}}$-ample, and 2) $R^{1}\left(\left.f\right|_{\left.U_{a^{\prime}}\right) *} \mathcal{O}\left(L^{* \otimes \mu}\right)\right.$ $=0$ for every $\mu>0$ on $U^{\prime} a^{\prime}$. As for 1) $a^{\prime}$ it is the direct consequence of [10, VIII, Théorèm 2.1], since $\left.L\right|_{f^{-1}\left(a^{\prime}\right)}$ is ample and $f$ is flat. If $A_{0}^{\prime}$ is a point, 2) also is well-known [10, VIII, Corollaire 1.4]. But Banica [2] proved the Grothendieck comparison theorem in the category of analytic spaces. Hence in our case we have the natural isomorphism

$$
\lim _{k} R^{1} f_{*}\left(L_{A, k}^{* \otimes \mu}\right)=R^{1} f_{*} L_{A}^{* \otimes \mu}
$$

where $\mathcal{Y}$ is the ideal sheaf of $A_{n}$ in $A, L_{A, k}^{* \otimes / n}=L_{A}^{* \otimes / n} / \mathcal{J}^{k} L_{A}^{* \otimes / n}$, and the right hand side is the $\mathcal{G}$-adic completion of $R^{1} f_{*} L_{A}^{* \otimes / \ell}$. Then the proof of $(2)$ can be carried through exactly as in the one point case. Thus we get $\left({ }^{*}\right)$. Next, put $U^{\prime}=\bigcup_{a^{\prime}} U_{a^{\prime}}^{\prime}$ and $U=f^{-1}\left(U^{\prime}\right)$. Then the property 1) and 2) holds if we replace $U_{a^{\prime}}$ by $U$, for, the properties are local with respect to $A^{\prime}$. Then we apply Theorem 2 to $U C W$ and $\left.f\right|_{U}: U \rightarrow U^{\prime}$, with $W$ a certain neighborhood of $U$ in $X$ such that $W \cap A=U$, and obtain a blowing down $\left(W^{\prime}, \tilde{f}\right)$ of $W$ along $\left.f\right|_{U}$ which may be considered to be over $S$ by Remark 3.

Q.E.D.

3.2. In this section we shall give examples which show that, in 
general the condition 2) of both Theorem 2 and Proposition 2 cannot be dropped. Before stating these, however, we stop to write down an explicit form of Theorem 2 (or Corollary) in certain simple cases.

Theorem $2^{\prime}$. Let $X$ be a complex manifold, $A$ a submanifold of $X$, and $f: A \rightarrow A^{\prime}$ a fiber bundle over a complex manifold $A^{\prime}$ with the typical fiber $F$ which is connected. Assume that

1) the restriction $\left.N_{A / X}\right|_{F}$ of the normal bundle of $A$ in $X$ to each fiber $F$ is weakly negative in the sense of Grauert [7], (or equivalently, the dual $\left.N_{A / X}^{*}\right|_{F}$ is ample).

2) $H^{1}\left(F,\left.N_{A / X}^{*(\mu)}\right|_{F}\right)=0$ for every $\mu>0$, where $N_{A \mid X}^{*\left(\mu^{\prime \prime}\right)}$ is the n-th symmetric product of $N_{A / X}^{*}$.

Then there exists a unique (except for isomorphisms) normal complex space $X^{\prime}$ and a proper surjective morphism $f^{\prime}: X \rightarrow X^{\prime}$ such that $\left(X^{\prime}, f^{\prime}\right)$ is the blowing down of $X$ along $f$.

Proof. It follows from 2) that $R^{1} f_{*} N_{A / X}^{*(\mu)}=0$ for $\mu>0$ and hence 2) implies the condition 2) of the corollary, since $\mathcal{G}_{A}^{\mu} / \mathcal{G}_{A}^{\mu+1}=N_{A / X}^{*(\mu)}$ as $\mathcal{O}_{A^{-}}$ modules. So we shall see that 1) implies the condition 1 ) of the corollary. Since $C_{X, A}=N_{A / X}$ in this case, we have to show that $N_{A / X}$ can be blowing down along $f$. Let $\sigma: \tilde{N}_{A / X} \rightarrow N_{A / X}$ be the blowing up with center $A$, the 0 -section of $N_{A / X}$, and $\tilde{A}=\sigma^{-1}(A)$. Then $\tilde{N}_{\tilde{A} / \tilde{X}}=N_{\tilde{A} / \tilde{X}}$ is (weakly) negative, and hence the dual $N_{\tilde{A} / \tilde{X}}^{*}$ is ample on each fiber of $f_{1}=f \cdot \sigma \mid \tilde{A}$ (c.f. Remark 1). Then $N_{\tilde{A} / \tilde{X}}^{*}$ is $f_{1}$-ample, since $f_{1}$ is smooth. Then the rest of the proof is the same as in that of Corollary to Theorem 2. Since in this case, the sheaf $\mathcal{S}_{X, A, f}$ defined by $(1)$ coincides with $\mathcal{O}_{X}$, the universal blowing-down $\left(X^{\prime}, f^{\prime}\right)$ of $X$ along $f$ is normal. This proves Theorem $2^{\prime}$.

By the vanishing theorem of Kodaira we have

Corollary. Let $X, A$, and $f: A \rightarrow A^{\prime}$ be as in Theorem 2'. Assume further that $A$ is of codimension 1 in $X$. If both $\left.N_{A / X}\right|_{F}$ and $\left.N_{A / X}\right|_{F}$ $\otimes K_{F}$ is negative (in any sense), then the conclusion of Theorem 2 holds, where $K_{F}$ is the canonical bundle of $F$.

Hence in particular, if $K_{F}$ is semi-negative (Def. 1), it suffices to 
assume only the negativity of $\left.N_{A / X}\right|_{F}$ to get the blowing-down.

Typical examples of such $F$ are projective space, abelian varieties, etc.

The above Theorem and Corollary can of course be formulated as well under the weaker assumption that $f$ is a smooth morphism (see [3]).

Now we proceed to give examples stated in the beginning of this section. Namely, we prove

Proposition 3. Let $F$ be a (connected) nonsingular projective varicty and $\pi: E \rightarrow F$ a vector bundle over $F$. Assume the following conditions;

1) $E$ is weakly negative, and

2) there exists an integer $\mu>0$ for which

$$
H^{1}\left(F, E^{*(\mu)}\right) \neq 0
$$

Then there exists a complex manifold $X$, a submanifold $A$ of $X$ and a fiber bundle $f: A \rightarrow A^{\prime}$ over a complex manifold $A^{\prime}$ with typical fiber $F$ with the following properties;

a) $\left.N_{A / X}\right|_{F} \cong E$, and

B) $X$ can never be blown down along $f$.

Proof. We only deal with the case that $E$ is a line bundle. The general case can then be treated roughly as follows; first let $\sigma: \widetilde{E} \rightarrow E$ be the blowing up with center the 0 -section $F_{0}$ of $E$ and let $\widetilde{F}_{0}=\sigma^{-1}\left(F_{0}\right)$. Apply the result for line bundles to the pair $\left(\widetilde{F}_{0}, N_{\tilde{F}_{0} / \tilde{E}}\right)$ to obtain manifolds $\widetilde{X}, \tilde{A}$, and $f: \tilde{A} \rightarrow A^{\prime}$ as in the Proposition. Then it is easy to see that $\tilde{f}$ factors as $\tilde{f}=f_{2} \cdot f_{1}, f_{1}: \tilde{A} \rightarrow A$ and $f_{2}: A \rightarrow A^{\prime}$, where $f_{1}$ (resp. $f_{2}$ ) is the fiber bundle with typical fiber $\mathbb{P}^{t}$ (resp. $F$ ). Then we blow down $\widetilde{X}$ along $f_{1}$ to a complex manifold $X$. This $X$ with $f_{2}: A \rightarrow A^{\prime}$ is seen to have the desired properties.

Now let $E$ be a line bundle over $F$. We denote $E$ by $Y$ when we consider the bundle space of $E$ as a complex manifold. Then we have a natural isomorphism

$$
H^{1}\left(Y, \mathcal{O}_{Y}\right) \cong \bigoplus_{\nu=0} H^{1}\left(F, E^{* \otimes 2}\right)
$$


where the right hand side is actually a finite sum, since $E^{*}$ is ample. Let $\xi^{\prime} \in H^{1}\left(F, E^{* \otimes \mu}\right)$ be a non-zero element and let $\xi \in H^{1}\left(Y, \mathcal{O}_{Y}\right)$ be the element corresponding to $\xi^{\prime}$ by the above isomorphism. Let $\pi: X \rightarrow Y$ be the affine bundle corresponding to $\xi$. Let $F_{0}$ be the 0 -section of $Y \rightarrow F$. Then $X$ is trivial as an affine bundle on every $\mu^{\prime}$-th neighborhood of $F_{0}$ if $\mu^{\prime}<\mu$. To see more explicitly, let $\left\{U_{\alpha}\right\}$ be a finite stein covering of $F$ such that $E$ is defined by the system of transition functions $\left\{f_{\alpha \beta}\right\}$ with respect to $\left\{U_{\alpha}\right\}$. Then $\xi^{\prime}$ is represented by a cocycle $\left\{\xi_{\alpha \beta}^{\prime}\right\}$ with respect to this covering. Then $\xi$ is represented in turn by the cocycle $\left\{\xi_{\alpha \beta}=\zeta_{\alpha}^{\mu} \xi_{\alpha \beta}^{\prime}\right\}$ with respect to the covering $V=\left\{V_{\alpha}=\pi^{-1}\left(U_{\alpha}\right) \cong U_{\alpha} \times C\right\}$ of $Y$, where $\zeta_{\alpha}$ is the fiber coordinate of $E \rightarrow F$. Then $X$ is a manifold defined as the union $X=\bigcup_{\alpha}\left(V_{\alpha} \times \mathbb{C}\right)$, where $\left(\left(P, \zeta_{\alpha}\right), \eta_{\alpha}\right) \in V_{\alpha} \times \mathbb{C}$ and $\left(\left(P, \zeta_{\beta}\right), \eta_{\beta}\right) \in V_{\beta} \times C$ are identified if and only if

$$
\left\{\begin{array}{l}
\eta_{\alpha}=\eta_{\beta}+\xi_{\alpha \beta}^{\prime} \zeta_{\alpha}^{\mu} \\
\zeta_{\alpha}=f_{\alpha \beta} \zeta_{\beta},
\end{array}\right.
$$

$\pi$ being defined by $\left(\left(P, \zeta_{\alpha}\right), \eta_{\alpha}\right) \rightarrow\left(P, \zeta_{\alpha}\right)$ on each $V_{\alpha} \times \mathbb{C}=\pi^{-1}\left(V_{\alpha}\right)$. Define $A$ to be $\pi^{-1}\left(F_{0}\right)$. Since $\zeta_{\alpha}=0$ is the local equation of $F_{0}$ on each $V_{\alpha}$, by (4) we infer that $A \cong F \times C$. Let $f: A \rightarrow C$ be the map induced by the projection to the second factor by this isomorphism. Since $A$ is defined by $\zeta_{\alpha}=0$ on $V_{\alpha} \times G$, we have $\left.N_{A / X}\right|_{F} \cong E$. This is $\alpha$ ). It remains to show that $X$ can never be blown down along $f$. Assume there exists a blowing down;

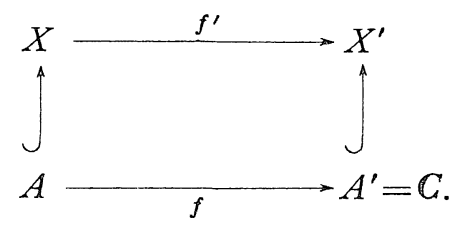

Let $\sigma: Y \rightarrow Y^{\prime}$ be the normal contraction of the 0 -section. Then we may take $\left(X^{\prime}, f^{\prime}\right)$ in such a way that $\exists \pi^{\prime}: X^{\prime} \rightarrow Y^{\prime}$ with $\pi^{\prime} f^{\prime}=\sigma \cdot \pi$. Indeed, we have only to take $X^{\prime}$ to be normal. Now take a point $P \in A^{\prime} \hookrightarrow X^{\prime}$ and let $S^{\prime}$ be a closed subspace of codimension 1 in $\left(\pi^{\prime}\right)^{-1}(V)$ such that $S^{\prime} \cap A^{\prime}=\{P\}$, where $V$ is a small neighborhood of $\sigma\left(F_{0}\right)$. Let $S$ be the 
proper transform of $S^{\prime}$ in $X$. Then taking $V$ sufficiently small we may assume that $S$ contains no fibers of $\pi$ (c.f. [16, I]). Suppose $S$ intersects with each fiber $X_{y}, y \in V$, at $n$ points counting with multiplicities (the number $n$ is independent of $y$ ), and $S_{1, y}, \ldots, S_{n, y}$, be those points over $y$. Then by taking the arithmetic mean $\frac{s_{1, y}+\ldots+s_{n, y}}{n}$ of $s_{i, y}$, which has the meaning since $X$ is an affine bundle (see [7]), we have a section $\bar{S}$ over $V$ of the nontrivial affine bundle $X$. This contradicts the fact that $\xi^{\prime} \neq 0$. In fact, using this section we can easily get the coboundary relation for $\xi^{\prime}$.

Q.E.D.

This provides a counterexample to the conjecture of [21], where it is said that the condition 1) will be sufficient to get the blowing-down in Theorem 2'.

On the other hand, it also furnishes an example of a complex space $Z$ which is not holomorphically convex, but whose reduction $Z_{\text {red }}$ is holomorphically convex (c.f. [28]). Indeed, we define $Z$ to be a complex space $\left(A, \mathcal{O}_{X} / \mathcal{G}^{\mu+1}\right)$, where the notations are as in the previous Proposition. Then $Z_{r e d}=A \cong F \times C$ is clearly holomorphically convex. But if $Z$ is holomorphically convex and $\tau: Z \rightarrow Z^{\prime}$ is the Remmert quotient of $Z$, then $Z_{r e d}^{\prime}=C$ and $\tau_{r e d}$ is isomorphic to $p_{2}$, the projection to the second factor. Then by the same argument as above we can get a contradiction. Finally, using the example of Proposition 3, we construct an example which shows that the conclusion of Proposition 3 does not continue to be true if the condition 2) is omitted. Hence, roughly, a deformation of a blowing-down is in general not a blowing-down.

Example. We use the notations of Proposition 3. Define $X_{t}, t \in \mathbb{C}$, to be affine bundles over $Y$ defined by

$$
\left\{\begin{array}{l}
\eta_{\alpha}=\eta_{\beta}+t \zeta_{\alpha}^{\mu} \xi_{\alpha \beta}^{\prime} \\
\zeta_{\alpha}=f_{\alpha \beta} \zeta_{\beta}
\end{array}\right.
$$

Then $X=\bigcup_{t \in C} X_{t}$ forms a family of affine bundles over $Y$ parametrized by $S=C$. We see easily that $X_{0} \cong Y \times C$ and $X_{t} \cong X_{1}$ if $t \neq 0$. Let $\pi_{t}: X_{t} \rightarrow Y$ be the projection and put $A_{t}=\pi_{t}^{-1}\left(F_{0}\right)$. Then we have the 
natural $S$-morphism $f: A=\cup A_{t} \rightarrow A^{\prime}=C \times S$. Since $f \mid A_{0}$ coincides with the projection to the first factor and $N_{A_{0} / X_{0} \mid F} \cong E$, we have $R^{1} f_{*}$ $N_{A 0 / X_{0}}^{*(\mu)} \neq 0$, though $N_{A_{0} / X_{0}}$ is negative. Now it is readily seen that the existence of a blowing down of $X$ along $f$ would lead to a contradiction with the non-existence of a blowing down of $X_{0}$ along $f_{0}$. Hence this $(X, A, f, S)$ is proved to be our example.

\section{References}

[1] Artin, M., Algebraization of formal moduli, Ann. of Nath., 91 (1970), 88-135.

[2] Banica, C., Le complete formel d'un espace analytique le long d'un sous-espace: Un theoreme de comparaison, Manuscripta Math., 6 (1972), 207-244.

[3] Cornalba, N., Two theorems on modifications of analytic spaces, Invent, Math., 20 (1973), 227-247.

[4] Fujiki, A. and Nakano, S., Supplement to "On the inverse of monoidal transformation", Publ. RIMS, Kyoto Univ., 7 (1971).

[5] Grauert, H., Bemerkenswerte pseudekonvexe Mannigfaltigkeiten. Math. Z., 81 (1963). 377-391.

[6] Grauert, H., On Levi's problem and the imbedding of real analytic manifolds, Ann. of Math., 68 (1958), 460-472.

[7] Grauert, H., Über Modifikationen und exzeptionelle analytische Mengen, Math. Ann., 146 (1962), 366-466.

[8] Griffiths, P., The extention problem in complex analysis II, Amer. J. Math., 88 (1966), 366-446.

[9] Grothendieck, A., Êlements de géométrie algébrique, Publ. Math. I.H.E.S., 8 (1961).

[10] Grothendieck, A.. Technique de construction en géométrie analytique I-X, Seminaire Cartan (1960/1).

[11] Hartshorne, R., Ample sulvarieties of algebraic varieties, Lecture Notes in Math., 156, Springer (1970).

[12] Hironaka, H.. Flattening by blowing up, Proc. Internat. Conf. on Manifolds and Related topics in topology (Tokyo, 1973), to appear.

[13] Hironaka, H., Formal line bundles along exceptional loci, Proc. of the Bombay Colloqium on Algebraic Geometry, (1968), 201-218.

[14] Hironaka, H. and Rossi, H., On the equivalence of imbeddings of exceptional complex spaces, Math. Ann., 156 (1964), 313-333.

[15] Hirzebruch, F., Topological methods in algebraic geometry, 3rd Ed., Berlin-HeidelbergNew York Springer (1966).

[16] Houzel, C., Géométrie analytique local, I II, Seminare Cartan (1960/1).

[17] Knorr, K. and Schneider, M., Relativexzeptionelle Analytische Nengen, Math. Ann. 193 (1971), 238-254.

[18] Kodaira, K., On Käler varieties of restricted type, Ann. of IMath., 60 (1954), 38-48.

[19] Lascu, A. T., Sous-variétés régulièrement contractible d'une variété algébrique, Annali Sci. Norm. Sup. di Pisa, 23 (1969), (675-695).

[20] Lojasiewicz, S., Sur le problème de la division, Studia 1Math., 18 (1959), 87-136.

[21] Markoe, A. and Rossi, H., Families of strongly pseudoconvex manifolds, Symp. on several complex variables, Park City, Utah, Lecture Notes in Math., 184, Springer (1970) 182-207. 
[22] Moisezon, B. G., On n-dimensional compact complex varieties with $\mathrm{n}$-algebraically independent meromorphic functions, AMS translations (2), 63 (1967), 51-177.

[23] Nakano, S., On the inverse of monoidal transformations, Publ. RIMS, Kyoto Cruiv., 6 (1970), 483-502.

[24] Nakano, S., Vanishing theorems for weakly 1-complete manifolds, Number theory, algebraic geometry and commutative algcbra, in honor of Y. Akisuki, Kinokuniya, (1973), 169179.

[25] Rossi, H., Picard variety of an isolated singular point, Rice univ. Studies, 54 (1968), 63-73.

[26] Siu, Y. T., The 1-convex generalization of Grauert's direct image theorem, Math. Ann., 190 (1971), 203-214.

[27] Wiegmann, K.-W.. Einbettungen komplexer Rảume in Zahlenräume, Invenl. Math., 1 (1966), 229-242.

[28] Uiegmann, K.-W., Über Quotienten holomorph-konvexer komplexer Räume, Math. Z., 97 (1967), 251-258. 
